\title{
The function of metformin in endometrial receptivity (ER) of patients with polycyclic ovary syndrome (PCOS): a systematic review and meta-analysis
}

\author{
Lifang Yuan ${ }^{1 \dagger}$, Hongbo Wu ${ }^{1,2 \dagger}$, Weiyu Huang ${ }^{1}$, Yin Bi ${ }^{1}$, Aiping Qin ${ }^{1}$ and Yihua Yang ${ }^{1 *}$
}

\begin{abstract}
Background: This meta-analysis summarizes evidence from studies using metformin (Met) to improve endometrial receptivity (ER) in women with PCOS.

Methods: Following the PRISMA protocol, we conducted a comprehensive search of academic literature from various databases, including PubMed, EMbase and Cochrane libraries. Studies published in English before Jan 27, 2021, were recruited for primary screening. Data on endometrial thickness (EMT), endometrial artery resistance index (RI), clinical pregnancy rate (CPR) and miscarriage rate (MR) were extracted and analyzed.

Results: Sixty-two eligible studies that included 6571 patients were evaluated in this meta-analysis. Primary indicators are EMT and endometrial aetery Rl; secondary indicators include the clinical pregnancy rate and miscarriage rate. Metformin significantly increased EMT $(S M D=2.04,95 \% \mathrm{Cl}(0.96,3.12), P=0.0002)$ and reduced endometrial artery RI compared to the non-Met group (SMD $=-2.83,95 \% \mathrm{Cl}:(-5.06,-0.59), P=0.01)$. As expected, metformin also improved CPR and reduced MR in PCOS patients as a result, clinical pregnancy rate (risk ratio $[\mathrm{RR}]=$ $1.26,95 \% \mathrm{Cl}: 1.11-1.43, P=0.0003)$, and miscarriage rate ( $\mathrm{RR}=0.73,95 \% \mathrm{Cl}: 0.58-0.91, P=0.006)$.

Conclusion: Metformin may improve endometrial receptivity (ER) in PCOS patients by increasing EMT and reducing endometrial artery Rl. However, the level of most original studies was low, with small sample sizes. More large-scale, long-term RCTs with rigorous methodologies are needed.
\end{abstract}

Keywords: Metformin, PCOS, Endometrial receptivity, Endometrial thickness, Meta-analysis

\section{Introduction}

During recent decades, assisted reproductive technologies (ARTs), such as IUI, in vitro fertilization (IVF) and embryo transfer (ET), have become increasingly popular. Move over, the prognosis of ART treatment for infertility patients has greatly improved; however, some

\footnotetext{
*Correspondence: workyyh@163.com

${ }^{+}$Lifang Yuan and Hongbo Wu contributed equally to this work.

${ }^{1}$ Reproductive Medical Center, the First Affiliated Hospital of Guangxi Medical University, Nanning, China

Full list of author information is available at the end of the article
}

patients still cannot achieve clinical pregnancy after receiving multiple high-quality embryo transfers. There are many causes that may explain such implantation failure, and endometrial receptivity (ER) is critical [1]. By definition, ER refers to the ability of the endometrium to allow the blastula to locate, adhere, invade, and ultimately implant during the period when the endometrium matures (luteal phase) [2]. A successful pregnancy requires synchronization of the development of the embryo and a receptive endometrium [3]. It is generally accepted that the quality of the embryo and ER play an

(c) The Author(s). 2021 Open Access This article is licensed under a Creative Commons Attribution 4.0 International License, which permits use, sharing, adaptation, distribution and reproduction in any medium or format, as long as you give appropriate credit to the original author(s) and the source, provide a link to the Creative Commons licence, and indicate if changes were made. The images or other third party material in this article are included in the article's Creative Commons licence, unless indicated otherwise in a credit line to the material. If material is not included in the article's Creative Commons licence and your intended use is not permitted by statutory regulation or exceeds the permitted use, you will need to obtain permission directly from the copyright holder. To view a copy of this licence, visit http://creativecommons.org/licenses/by/4.0/. The Creative Commons Public Domain Dedication waiver (http://creativecommons.org/publicdomain/zero/1.0/) applies to the data made available in this article, unless otherwise stated in a credit line to the data. 


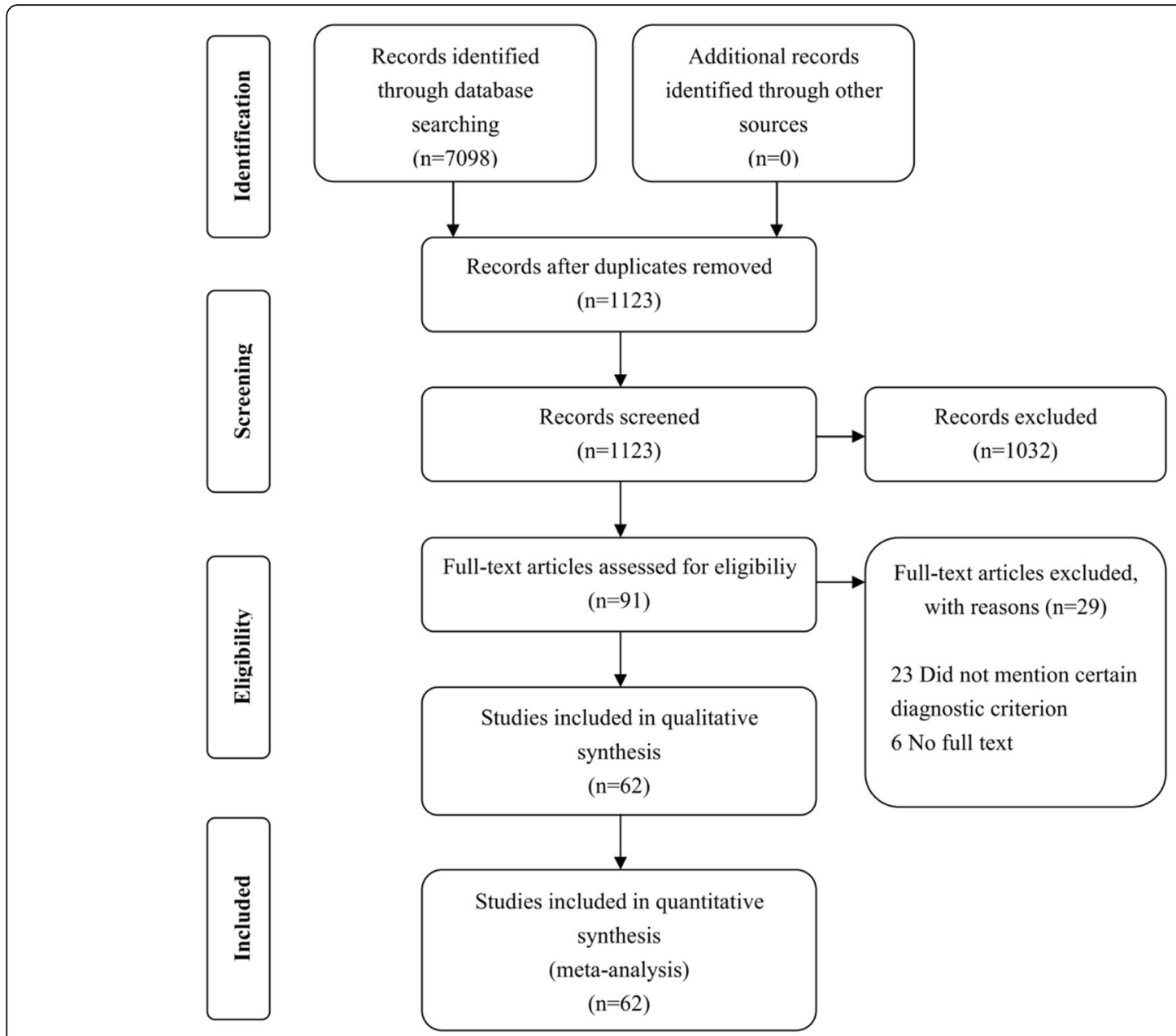

Fig. 1 PRISMA flow diagram of study selection

Table 1 Baseline characteristics of the included studies

\begin{tabular}{|c|c|c|c|c|c|c|}
\hline No & Study & Intervention & Rescearch type & \begin{tabular}{|l|l} 
Number \\
\end{tabular} & Years(I/C) & Related indicators \\
\hline 1 & DANIELA 2001 & Met $500 \mathrm{mg}$ Tid & ccs & 52 & $27 \pm 1 / 27 \pm 1$ & EMT, RI \\
\hline 2 & David T 2001 & Met $500 \mathrm{mg}$ Tid & $\operatorname{ccs}$ & 25 & $29+1.230 \pm 1.0$ & CPR \\
\hline 3 & Korak 2002 & Met $800 \mathrm{myg}$ Bid & $\cos$ & 55 & $30.47 \div 5.2531 .11 \pm 3.5$ & EMT,PCR \\
\hline 4 & Sturrock 2002 & Met $500 \mathrm{mg}$ Tid & $\cos$ & 26 & $18-40 / 18-40$ & PCR \\
\hline 5 & Malkawi 2003 & Met $850 \mathrm{mg}$ Bid & $\operatorname{ccs}$ & 161 & $23-3423-34$ & CPR,MR \\
\hline 6 & Kjotrod 2004 & Met $500 \mathrm{mg}$ Bid & $\operatorname{ccs}$ & 27 & $29.0-31.5 / 528.7-732.7$ & CPR,MR \\
\hline 7 & Liu 2004 & Met $500 \mathrm{mg}$ Tid & $\operatorname{ces}$ & 50 & Not Mentioned & CPR \\
\hline 8 & Palomba 2004 & Met 850mg Bid & RCT & 109 & 26.842 .227 .542 .4 & CPR.MR \\
\hline 9 & Sathin 2004 & Met 850mg Bid & $\operatorname{ccs}$ & 21 & $21-31 / 19-28$ & CPR \\
\hline 10 & Tasulenit 2005 & Met 850my Bid & $\operatorname{ccs}$ & 32 & $30.6 \pm 3.231 .8 \pm 2.7$ & CPR.EMT \\
\hline 11 & Hwu 2005 & Met S00mg Tid & RCT & 80 & $29.07 \pm 4.45 / 27.80+3.75$ & CPR \\
\hline 12 & Palomba 12005 & Met $850 \mathrm{mg}$ Bid & RCT & 70 & $26.6+2.7726 .922 .8$ & CPR.MR \\
\hline 13 & Palomba II 2005 & Met $850 \mathrm{mg}$ Bid & $\operatorname{ccs}$ & 92 & $20-34 / 20-34$ & CPR,MR \\
\hline 14 & Palomba III 2005 & Met 850mg Bid & RCT & 28 & $22-3422-34$ & CPR.MR \\
\hline 15 & Moll 2006 & Met $500 \mathrm{mg}$ Tid & RCT & 162 & $27.9 \pm 3.728 .444 .7$ & CPR.MR \\
\hline 16 & Nestler 2006 & Met 850mg Bid & RCT & 92 & $20-34 / 20.34$ & CPR,MR \\
\hline 17 & Palomba 2006 & Met $850 \mathrm{mg}$ Bid & RCT & 74 & $25.8 \pm 2.326 .3 \pm 3.0$ & RI, EMT \\
\hline 18 & Tang 2006 & Met $850 \mathrm{mg}$ Bid & RCT & 101 & $20-3920-39$ & CPREMT \\
\hline 19 & Karimzadeh 2007 & Met S50omg Tid & RCT & 200 & $20-35 / 20-35$ & CPR \\
\hline 20 & Neveu 2007 & Met $500 \mathrm{mg}$ Tid & RCT & 113 & $29.3 \div 0.5 / 29.110 .5$ & CPR.MR \\
\hline 21 & Palomba 2007 & Met 850mg Bid & cs & 72 & $22-4020-41$ & CPR.MR \\
\hline 22 & Richard 2007 & Met $50 \mathrm{omg}$ Tid & RCT & 418 & $28.3 \pm 4.027 .9 \pm 4.0$ & CPR \\
\hline 23 & Schachter 2007 & Met $850 \mathrm{mmg}$ Bid & RCT & 51 & $28.8 \pm 0.428 .8+0.4$ & CPR,MR \\
\hline 24 & Moll 2007 & Met $500 \mathrm{mg}$ Tid & RCT & 225 & $27.9 \pm 3.7 / 28.4 \pm 4.7$ & CPR \\
\hline 25 & Wei 2008 & Met $500 \mathrm{mg}$ Bid & RCT & 54 & $28.642 .5 / 28.6 \pm 3.3$ & CPR,MR \\
\hline 26 & Kazstooni 2009 & Met S00mg Tid & RCT & 37 & $17-3517-35$ & CPR,EMT \\
\hline 27 & Qublan 2009 & Met $850 \mathrm{mg}$ Bid & RCT & 66 & $34.6+4.3 / 333.8+3.9$ & CPR.MR \\
\hline 28 & Zain 2009 & Met $50 \mathrm{omg}$ Tid & RCT & 77 & $29.314 .95 / 29.6614 .35$ & CPR \\
\hline 29 & Abu Hashim I 2010 & Met $501 \mathrm{mg}$ Tid & RCT & 192 & $26.8 \pm 2.227 .3+2.6$ & CPR.MR,EMT \\
\hline 30 & Abu Hashim II 2010 & Met $500 \mathrm{mg}$ Tid & RCT & 127 & $262 * 2.226 .2+2.2$ & EMT \\
\hline 31 & Baran 2010 & Met 850mg Bid & $\cos$ & 61 & $25: 5 \pm 4.3627 .35 \div 399$ & CPR,MR \\
\hline
\end{tabular}

\begin{tabular}{|c|c|c|c|c|c|c|}
\hline No & Study & Interrention & Research type & Number & Vearn(T/C) & Related indicators \\
\hline 32 & Brewer 2010 & Met $850 \mathrm{mg}$ Bid & RCT & 142 & $31 / 32$ & CPR,MR \\
\hline 33 & J.Cheng 2010 & Met 500mg Tid & RCT & 45 & $27.00+2.992 .70+3.13$ & CPR \\
\hline 34 & Jahnson 2010 & Met 500my Tid & RCT & 71 & 2924.72824 .0 & CPR,MR \\
\hline 35 & Karimzadeh 2010 & Me s00omg Tid & RCT & 178 & $27.34+22727,47+2.38$ & CPR \\
\hline 36 & Palomba I 2011 & Met $850 \mathrm{mg}$ Bid & RCT & 257 & $275 \pm 4.4282+4.3$ & CPR,MR \\
\hline 37 & Abu Hashim I 2011 & Met S00mg Tid & RCT & 138 & $27.2+2.5 / 26.5+2.3$ & CPR,MR,EMT \\
\hline 38 & Abu Hashim II 2011 & Met 500mg Tid & RCT & 75 & $27.5+2.426 .8+2.2$ & EMT \\
\hline 39 & Johnson 2011 & Met S00ang Tid & RCT & 64 & Not Mentioned & CPR \\
\hline 40 & Kjotrod 2011 & Met 500mg Tid & RCT & 149 & $29.6 \pm 3.429 .5 \pm 3.8$ & CPR \\
\hline 41 & Palomba II 2011 & Met $500 \mathrm{mg}$ Tid & RCT & 120 & $21-3 y / 22.34$ & CPR,MR \\
\hline 42 & Swanton 2011 & Met S00mg Tid & RCT & 134 & $32.0+3.7 / 32.9+3.9$ & CPR \\
\hline 43 & Basirat 2012 & Met Solmg Tid & RCT & 334 & $24.86+3.7825 .26+4.32$ & CPR \\
\hline 44 & Morin 2012 & Met SOOMg Tid & RCT & 320 & $28.4+3.925 .3422 .3$ & CPR,MR \\
\hline 45 & Ayaz 2013 & Met 500mg Tid & RCT & 42 & $32 \pm 3.5 / 31,3 \pm 2.9$ & CPR \\
\hline 46 & Mosummat 2013 & Met $500 \mathrm{mg}$ Tid & RCT & 96 & $27.154+2026964.05$ & CPR.MR \\
\hline 47 & Hosscini 2013 & Met 500 ang Tid & RCT & 368 & $26.92+3 \times 2 \times 26.71+3.73$ & CPR \\
\hline 48 & Kar 2013 & Met 500mg Tid & $\operatorname{ccs}$ & 61 & Not Mentiones & CPR \\
\hline 49 & Mohsen 2013 & Met 85 ong Bid & PS & so & 2546025463 & EMT,RI \\
\hline so & An.Y. 2014 & Met Soomg Tid & RCT & 72 & $28.2+3.828 .2+3.8$ & CPR,EMT \\
\hline 51 & Kumar 2014 & Met $850 \mathrm{mg}$ Bid & $\operatorname{ccs}$ & 60 & $<40<40$ & CPR \\
\hline 52 & Vahidin 2014 & Met $850 \mathrm{mg}$ Bid & $\operatorname{ccs}$ & 20 & $435<35$ & CPR.EMT \\
\hline 53 & Fermander 2015 & Met $500 \mathrm{mg}$ Tid & RCT & 34 & $26: 9,4 / 28: 05$ & CPR \\
\hline 54 & Maged 2015 & Met $500 \mathrm{ming}$ Tid & RCT & 80 & $25.8+3.5 / 26.0+3.6$ & CPR,EMT \\
\hline 55 & Wang 2015 & Met $850 \mathrm{mg}$ Bid & RCT & 110 & $23.6 \pm 2.624 .3+4.5$ & CPR,MR \\
\hline 56 & Cheraghi 2016 & Met $500 \mathrm{mg}$ Tid & RCT & 30 & $28.07+3,41 / 27,9+2.8$ & CPR,EMT \\
\hline 57 & Jacob 2016 & Met 85 Somg Bid & RCT & 153 & $29.9 \pm 4,429.6 \pm 3.9$ & CPR \\
\hline 58 & Liu 2017 & Met 500min Tid & RCT & 28 & $27.2+2826.8 \pm 3.1$ & CPR.MR \\
\hline 59 & Abdalmagced 2019 & Met 500mg Bid & RCT & 102 & $31.1+3.731 .89+3.6$ & CPR,MR \\
\hline 60 & Jiang 2019 & Met 2500 perd & RCT & 79 & $27.46 \pm 3.4 \times 26.87 \pm 4.01$ & CPR,MR \\
\hline 61 & Pourghasem 2019 & Met 500mg Tid & RCT & 100 & $31.06+1.11 / 30.42+2.58$ & CPR \\
\hline 62 & Prabhakar 2020 & Met soomg Tid & & & $28.2 \times+3.4127 .86+3.06$ & CPR: \\
\hline
\end{tabular}

Abbreviation: $R C T$ randomized controlled trial, CS cohort study, CCS case-control study, PS prospective study, MET metformin, CC clomiphene, CPR clinical pregnancy rate, $E M T$ endo metrial thickness, $M R$ miscarriage rate; $R /$ resistance index, Bid bis in die, Tid ter in die 
Table 2 Methods and results of the meta-analysis

\begin{tabular}{|c|c|c|c|c|c|c|c|c|c|}
\hline \multirow[t]{2}{*}{ Included studies } & \multicolumn{4}{|l|}{ Selection } & \multirow[t]{2}{*}{ Comparability } & \multicolumn{3}{|l|}{ Outcome } & \multirow{2}{*}{$\begin{array}{l}\text { Total quality } \\
\text { socres }\end{array}$} \\
\hline & $\begin{array}{l}\text { Representativeness } \\
\text { of the } \\
\text { exposed cohort }\end{array}$ & $\begin{array}{l}\text { Selection of } \\
\text { the } \\
\text { nonexposed } \\
\text { cohort }\end{array}$ & $\begin{array}{l}\text { Ascertainment } \\
\text { of exposure }\end{array}$ & $\begin{array}{l}\text { Demonstration } \\
\text { that outcome } \\
\text { of interest was } \\
\text { not present at } \\
\text { start of study }\end{array}$ & & $\begin{array}{l}\text { Assessment } \\
\text { of outcome }\end{array}$ & $\begin{array}{l}\text { Was } \\
\text { follow-up } \\
\text { long enough } \\
\text { for outcomes } \\
\text { to occur }\end{array}$ & $\begin{array}{l}\text { Adequacy } \\
\text { of } \\
\text { follow-up } \\
\text { of cohorts }\end{array}$ & \\
\hline DANIELA J 2001 & $\star$ & $\star$ & $\star$ & & $\star \star$ & $\star$ & & & 6 \\
\hline David T 2001 & $\star$ & $\star$ & $\star$ & & $\star$ & $\star$ & $\star$ & & 6 \\
\hline Kocak 2002 & $\star$ & & $\star$ & & $\star$ & $\star$ & $\star$ & & 5 \\
\hline Sturrock 2002 & $\star$ & & $\star$ & & $\star$ & $\star$ & & & 4 \\
\hline Malkawl 2003 & $\star$ & $\star$ & & & & $\star$ & & & 3 \\
\hline Kjotrod 2004 & $\star$ & $\star$ & $\star$ & & $\star \star$ & $\star$ & & & 6 \\
\hline Liu 2004 & $\star$ & $\star$ & $\star$ & & & $\star$ & & & 4 \\
\hline Sahin 2004 & $\star$ & & $\star$ & & $\star$ & $\star$ & & & 4 \\
\hline Tasdemir 2005 & $\star$ & $\star$ & $\star$ & & $\star \star$ & & & & 5 \\
\hline Palomba I 2005 & $\star$ & $\star$ & & & $\star$ & $\star$ & & & 4 \\
\hline Palomba II 2007 & $\star$ & $\star$ & $\star$ & & $\star \star$ & $\star$ & & & 6 \\
\hline Baran 2010 & $\star$ & $\star$ & & $\star$ & $\star$ & & & & 4 \\
\hline Kar 2013 & $\star$ & $\star$ & $\star$ & $\star$ & $\star$ & & & & 5 \\
\hline Mohsen 2013 & $\star$ & $\star$ & $\star$ & & & $\star$ & & & 4 \\
\hline Kumar 2014 & $\star$ & & $\star$ & & & $\star$ & & & 3 \\
\hline Vahidin 2014 & $\star$ & $\star$ & $\star$ & $\star$ & $\star \star$ & $\star$ & & & 7 \\
\hline
\end{tabular}

$\mathrm{Cl}$ confidence interval, $C C$ clomiphenecitrate, $L O D$ laparoscopic ovarian diathermy, $L$ letrozole, $M E T$ metformin, $M-H$ mantel-haenszel, $M D$ mean difference, $N A C N$ acetyl-cysteine, OR oddsratio, Std standard deviation

${ }^{\text {a }}$ Statistically significant difference

important role in pregnancy establishment and maintenance. However, improving ER remains a challenge for clinicians [4], and it is an crucial strategy to improve the live birth rate [5].

As an insulin sensitizer, metformin has been widely used in infertility clinics. It is generally applied to reduce insulin resistance and glucose metabolism abnormalities in PCOS patients [6]. To improve the pregnancy rate of PCOS patients, metformin has been widely used alone or in combination with clomiphene for ovulation induction [7], though the impact of ER on PCOS patients is still controversial.

Recently, many meta-analyses and systematic reviews have been conducted on women with infertility and PCOS treated with metformin, such as the ovulation rate, pregnancy rate, miscarriage rate, serum sex hormone levels, and adverse effects [8]. Nonetheless, few meta-analyses and systematic reviews have addressed the effects of metformin on ER in PCOS patients. The main purpose of this study was a comprehensive systematic review and meta-analysis to compare the effects of metformin used alone or in combination on ER in PCOS patients.

\section{Methods}

\section{Search strategy}

Strictly following the Preferred Reporting Items for Systematic Reviews and Meta-analyses (PRISMA) 2009 Checklist Protocol, two independent reviewers (Y.B. and W.Y.H) performed a literature search in three electronic databases (PubMed, EMbase, and Cochrane Library). Studies published before May 31, 2020, were retrieved using the following key words: "Metformin" [Mesh] OR Dimethylbiguanidine OR Dimethylguanylguanidine OR Glucophage OR Metformin Hydrochloride OR Hydrochloride, Metformin OR Metformin $\mathrm{HCl}$ OR $\mathrm{HCl}$, Metformin AND ("polycystic ovary syndrome" [Mesh] OR Ovary Syndrome, Polycystic OR Syndrome, Polycystic Ovary OR Stein-Leventhal Syndrome OR Stein Leventhal Syndrome OR Syndrome, Stein-Leventhal OR Sclerocystic Ovarian Degeneration OR Ovarian Degeneration, Sclerocystic OR Sclerocystic Ovary Syndrome OR Polycystic Ovarian Syndrome OR Ovarian Syndrome, Polycystic OR Polycystic Ovary Syndrome 1 OR Sclerocystic Ovaries OR Ovary, Sclerocystic OR Sclerocystic Ovary). Preselected results were limited to English publications. Manual searches were also conducted to acquire potentially eligible articles that might have been missed by computer-based searches.

\section{Study selection}

Two investigators (W.Y.H and Y.B) reviewed the titles and abstracts of all literatures identified by the search strategy to generate a list of relevant articles, and the full texts were searched and read by another two reviewers (L.F.Y and Y.X.Z). Any disagreement was resolved by discussion or based on the judgment of a third expert (Y.H.Y) until a consensus was reached.

\section{Eligibility criteria}

Studies were considered eligible for the meta-analysis and systematic review if they met the following inclusion criteria: (i) experimental or observational studies; (ii) subjects received treatment alone or in combination, and 


\begin{tabular}{|c|c|c|c|c|c|c|c|c|c|c|c|c|c|c|c|c|c|c|c|c|c|c|}
\hline \multicolumn{23}{|c|}{$(\mathrm{A}-1)$} \\
\hline 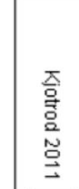 & 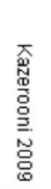 & 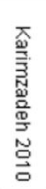 & 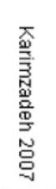 & 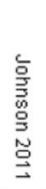 & 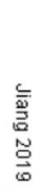 & 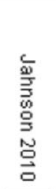 & 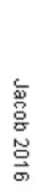 & $\begin{array}{l}\text { T } \\
\text { 空 } \\
\text { 宮 }\end{array}$ & 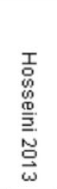 & 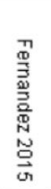 & 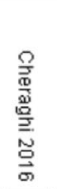 & 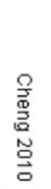 & 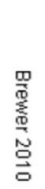 & 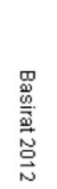 & 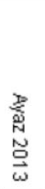 & 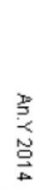 & 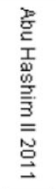 & 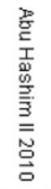 & 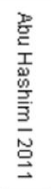 & 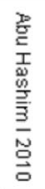 & 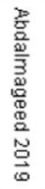 & \\
\hline$\odot$ & $\odot$ & $\odot$ & $\odot$ & $\odot$ & $\odot$ & $\odot$ & $\odot$ & $\sim$ & $\odot$ & $\odot$ & + & $\odot$ & $\oplus$ & $\odot$ & $\odot$ & $\odot$ & $\odot$ & $\odot$ & $\odot$ & $\odot$ & $\odot$ & Random sequence generation (selection bias) \\
\hline$\odot$ & (1) & $\odot$ & $\odot$ & $\odot$ & $\odot$ & $\odot$ & $\odot$ & $\odot$ & $\odot$ & $\odot$ & + & (1) & $\odot$ & $\odot$ & $\odot$ & $\odot$ & $\odot$ & $\odot$ & $\odot$ & $\odot$ & $\odot$ & Allocation concealment (selection bias) \\
\hline$\odot$ & $\odot$ & $v$ & $\odot$ & $\sim$ & $\odot$ & $\sim$ & $\odot$ & $\odot$ & $\odot$ & $\sim$ & $\sim$ & $\odot$ & $\odot$ & $\odot$ & $\odot$ & $\odot$ & $\sim$ & $\odot$ & $\sim$ & $\odot$ & $\sim$ & Blinding of participants and personnel (performance bias) \\
\hline$\odot$ & $\sim$ & $v$ & $\sim$ & (1) & (1) & $\sim$ & $\odot$ & $\sim$ & (1) & $\sim$ & $\sim$ & 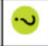 & $\sim$ & $\sim$ & $\sim$ & $\sim$ & $\odot$ & $\sim$ & $\odot$ & 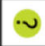 & $\odot$ & Blinding of outcome assessment (detection bias) \\
\hline (1) & $\odot$ & $\odot$ & (1) & $\odot$ & $\sim$ & $\sim$ & $\cdot$ & $\bullet$ & $\odot$ & $\odot$ & (1) & $\odot$ & (1) & $\odot$ & (1) & $\odot$ & (1) & $\odot$ & (1) & (1) & $\odot$ & Incomplete outcome data (attrition bias) \\
\hline$\odot$ & $\odot$ & $\odot$ & $\odot$ & $\odot$ & $\odot$ & $\odot$ & $\odot$ & $\odot$ & $\odot$ & $\odot$ & $\odot$ & 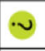 & $\sim$ & ( & $\odot$ & $\odot$ & $\odot$ & $\sim$ & $\odot$ & $\odot$ & 2 & Selective reporting (reporting bias) \\
\hline+ & $\odot$ & $\odot$ & $\odot$ & (1) & $\odot$ & $\odot$ & $\odot$ & $v$ & $\odot$ & + & + & (1) & + & + & $\odot$ & 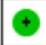 & + & 0 & 0 & + & + & Other bias \\
\hline
\end{tabular}

\section{(A-2)}

\begin{tabular}{|c|c|c|c|c|c|c|c|c|c|c|c|c|c|c|c|c|c|c|c|c|c|c|}
\hline 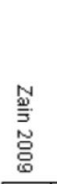 & $\begin{array}{l}\frac{\mathbf{s}}{N} \\
\text { 宮 }\end{array}$ & $\begin{array}{l}\text { s } \\
\text { 壳 } \\
N \\
\stackrel{O}{O} \\
\text { U. }\end{array}$ & $\begin{array}{l}\overrightarrow{-1} \\
\stackrel{\Xi}{\Xi} \\
\stackrel{0}{0} \\
\stackrel{亏}{\sigma}\end{array}$ & 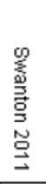 & 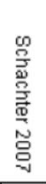 & 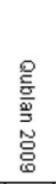 & 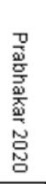 & 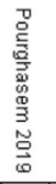 & 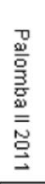 & 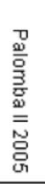 & 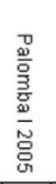 & 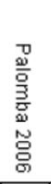 & 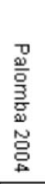 & 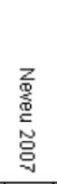 & 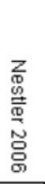 & 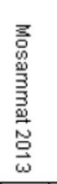 & $\begin{array}{l}\text { 言 } \\
\overline{3} \\
\text { 品 } \\
\text { N }\end{array}$ & 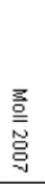 & $\begin{array}{l}\text { 흥 } \\
\bar{N} \\
\text { 宮 }\end{array}$ & 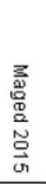 & $\begin{array}{l}\bar{\Sigma} \\
\text { N } \\
\stackrel{\Xi}{v}\end{array}$ & \\
\hline$\odot$ & $\odot$ & $\odot$ & $\odot$ & $\odot$ & $\odot$ & $\odot$ & 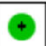 & 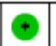 & 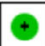 & $\odot$ & $\odot$ & 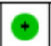 & $\odot$ & 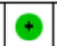 & $\odot$ & 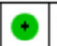 & + & $\bullet$ & $\odot$ & 0 & $\odot$ & Random sequence generation (selection bias) \\
\hline$\odot$ & $\odot$ & $\odot$ & $\sim$ & $\odot$ & $\odot$ & 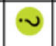 & $\odot$ & $\odot$ & $\odot$ & $\sim$ & $\odot$ & $\odot$ & $\odot$ & $\odot$ & $v$ & 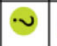 & $\odot$ & $\odot$ & $\odot$ & $\sim$ & $\sim$ & Allocation concealment (selection bias) \\
\hline$\odot$ & $\odot$ & $\odot$ & (1) & $\odot$ & $\sim$ & $\odot$ & $\sim$ & $\odot$ & 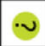 & $\odot$ & $\odot$ & $v$ & $\sim$ & $\odot$ & $\odot$ & $\odot$ & $\odot$ & $\odot$ & $\odot$ & $\odot$ & $\odot$ & Blinding of participants and personnel (performance bias) \\
\hline$\sim$ & 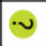 & (1) & $\cdot v$ & $\sim$ & $\cdot$ & $\sim$ & $\odot$ & $\cdot$ & $\cdot$ & 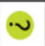 & $v$ & $\odot$ & 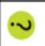 & 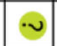 & $\odot$ & + & $\bullet$ & $\odot$ & $\cdot v$ & $\sim$ & $\odot$ & Blinding of outcome assessment (detection bias) \\
\hline 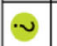 & $\odot$ & 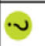 & $\odot$ & (?) & $\odot$ & (1) & $\odot$ & (1) & $\odot$ & $\odot$ & $\odot$ & $\sim$ & $\odot$ & $\odot$ & (1) & (1) & $\odot$ & $\odot$ & $\odot$ & (1) & (1) & Incomplete outcome data (attrition bias) \\
\hline (1) & $\odot$ & $\odot$ & $\odot$ & $\odot$ & $\odot$ & $\odot$ & $\sim$ & $\odot$ & + & $\odot$ & $\odot$ & $\odot$ & $\odot$ & 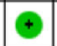 & $\odot$ & + & $v$ & $\odot$ & $\cdot v$ & $\odot$ & $\sim$ & Selective reporting (reporting bias) \\
\hline$\odot$ & $\cdot v$ & (1) & $\odot$ & $\odot$ & $\odot$ & $\bullet$ & $\odot$ & $\odot$ & $\odot$ & $\odot$ & $\odot$ & $\odot$ & $\odot$ & $\odot$ & $\odot$ & + & $\odot$ & $\odot$ & $\odot$ & $\bullet$ & $\odot$ & Other bias \\
\hline
\end{tabular}

(B)

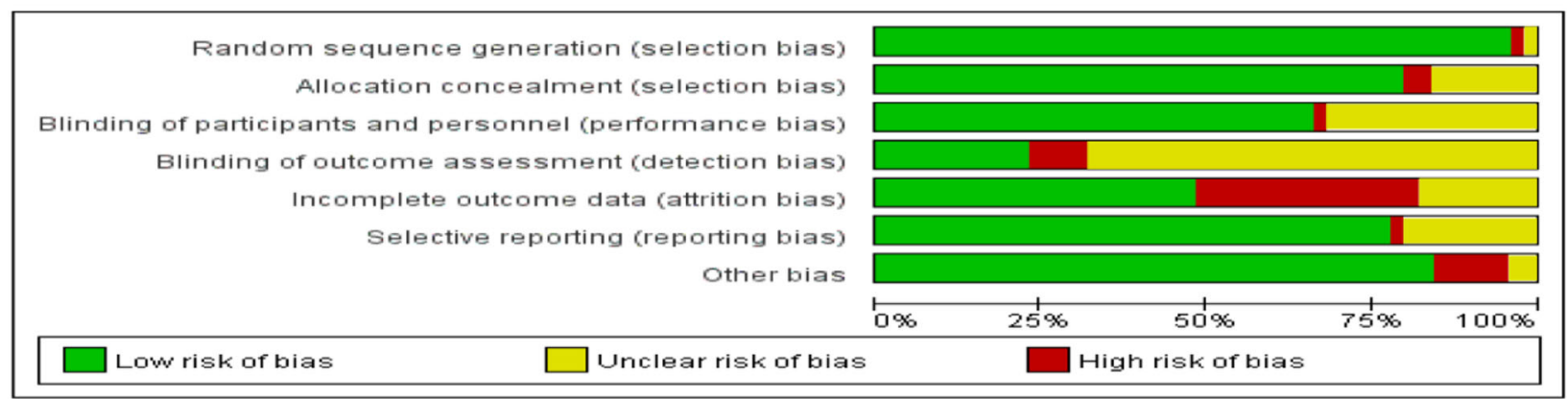

Fig. 2 Risk of bias for eligible randomized controlled trials

indicators related to ER were assessed. The exclusion criteria were as follows: (i) case reports and reviews; (ii) patients with endometrial hyperplasia, endometrial cancer, uterine myoma, endometrial polyps, intrauterine adhesion (IUA), and other pathological changes that could affect ER; (iii) studies published in non-English languages; and (iv) the full text could not access. Data were extracted from eligible studies by two reviewers using piloted screening forms in Microsoft Office Excel. Including the author, year of publication, number of participants, mean age, intervention, and time to measure EMT. The details of each outcome measure, such as EMT, endometrium artery RI, clinical pregnancy rate, and miscarriage rate, were precisely recorded.

\section{Statistical analysis}

The meta-analysis was conducted using Cochrane Review Manager software (RevMan 5.3). Continuous outcomes were measured by Std mean differences (SMDs) and dichotomous outcomes by risk ratios (RRs), both 
Table 3 Quality assessment of the included studies

\begin{tabular}{|c|c|c|c|c|c|}
\hline Outcome /subgroup title & $\begin{array}{l}\text { No. of } \\
\text { studies }\end{array}$ & $\begin{array}{c}\text { No. of } \\
\text { participants }\end{array}$ & Statistical method & Effect size & p-value \\
\hline 1.Endometrial thickness (EMT) & 13 & 1253 & Std(SMD IV,Random,95\%CI) & $2.04[0.96,3.12]$ & $0.0002^{\mathrm{a}}$ \\
\hline 1.1.1 Met Treatment After vs Before & 3 & 226 & Std(SMD IV,Random,95\%CI) & $1.95[0.33,3.75]$ & 0.02 \\
\hline 1.1.2 Met vs Placebo & 2 & 102 & Std(SMD IV,Random,95\%CI) & $0.24[-0.16,0.64]$ & 0.24 \\
\hline 1.1.3 Met+CC vs CC & 3 & 112 & Std(SMD IV,Random,95\%CI) & $1.15[0.62,1.68]$ & $<0.0001^{\mathrm{a}}$ \\
\hline 1.1.4 Met+CC Treatment After vs Before & 3 & 680 & Std(SMD IV,Random,95\%CI) & $5.10[2.24,7.97]$ & $0.0005^{\mathrm{a}}$ \\
\hline 1.1.5 Met+X vs X & 2 & 133 & Std(SMD IV,Random,95\%CI) & $0.49[0.14,0.83]$ & $0.006^{a}$ \\
\hline 2.Endometrium artery $\mathbf{R I}$ & 3 & 278 & Std(SMD IV,Random, $95 \% \mathrm{CI}$ ) & $-2.83[-5.06,-0.59]$ & $0.01^{\mathrm{a}}$ \\
\hline 2.1.1 Met Treatment After vs Before & 2 & 174 & Std(SMD IV,Random,95\%CI) & $-1.39[-4.09,1.31]$ & 0.31 \\
\hline 2.1.2 Met+CC Treatment After vs Before & 1 & 52 & Std(SMD IV,Random,95\%CI) & $-5.41[-6.62,-4.20]$ & $<0.00001^{\mathrm{a}}$ \\
\hline 2.1.3 Met+CC vs CC & 1 & 52 & Std(SMD IV,Random,95\%CI) & $-3.28[-4.14,-2.43]$ & $<0.00001^{\mathrm{a}}$ \\
\hline 3.Clinical pregnancy rate (CPR) & 56 & 6163 & $\mathrm{RR}(\mathrm{M}-\mathrm{H}$, Random, $95 \% \mathrm{CI})$ & $1.26[1.11,1.43]$ & $0.0003^{\mathrm{a}}$ \\
\hline 3.1.1 Met vs Placebo & 15 & 1753 & $\mathrm{RR}(\mathrm{M}-\mathrm{H}, \mathrm{Random}, 95 \% \mathrm{Cl})$ & $1.37[1.08,1.74]$ & $0.01^{\mathrm{a}}$ \\
\hline 3.1.2 Met vs CC & 6 & 359 & $\mathrm{RR}(\mathrm{M}-\mathrm{H}$, Random, $95 \% \mathrm{CI})$ & $1.40[1.11,1.76]$ & $0.004^{a}$ \\
\hline 3.1.3 Met+CC vs CC & 22 & 2305 & $\mathrm{RR}(\mathrm{M}-\mathrm{H}, \mathrm{Random}, 95 \% \mathrm{CI})$ & $1.39[1.15,1.69]$ & $0.0008^{\mathrm{a}}$ \\
\hline 3.1.4 Met+FSH vs FSH & 3 & 298 & $\mathrm{RR}(\mathrm{M}-\mathrm{H}, \mathrm{Random}, 95 \% \mathrm{CI})$ & $1.37[0.98,1.91]$ & 0.06 \\
\hline 3.1.5 Met+CC vs LOD & 7 & 981 & $\mathrm{RR}(\mathrm{M}-\mathrm{H}, \mathrm{Random}, 95 \% \mathrm{Cl})$ & $0.79[0.49,1.27]$ & 0.34 \\
\hline 3.1.6 Met+X vs X & 3 & 567 & RR(M-H,Random, $95 \% \mathrm{CI})$ & $1.21[0.97,1.50]$ & 0.10 \\
\hline 4. Miscarriage rate (MR) & 27 & 1362 & RR(M-H,Random, 95\%CI) & $0.73[0.58,0.91]$ & $0.006^{\mathrm{a}}$ \\
\hline 4.1.1 Met vs Placebo & 8 & 349 & $\mathrm{RR}(\mathrm{M}-\mathrm{H}, \mathrm{Random}, 95 \% \mathrm{CI})$ & $0.78[0.53,1.15]$ & 0.22 \\
\hline 4.1.2 Met vs CC & 5 & 203 & $\mathrm{RR}(\mathrm{M}-\mathrm{H}, \mathrm{Random}, 95 \% \mathrm{CI})$ & $0.51[0.26,0.99]$ & 0.05 \\
\hline 4.1.3 Met+CC vs CC & 4 & 227 & $\mathrm{RR}(\mathrm{M}-\mathrm{H}$, Random, $95 \% \mathrm{Cl})$ & $0.91[0.41,1.98]$ & 0.80 \\
\hline 4.1.4 Met+FSH vs FSH & 2 & 78 & RR(M-H,Random, $95 \% \mathrm{CI})$ & $0.70[0.18,2.67]$ & 0.60 \\
\hline 4.1.5 Met+CC vs LOD & 6 & 432 & $\mathrm{RR}(\mathrm{M}-\mathrm{H}$, Random, $95 \% \mathrm{CI})$ & $0.77[0.47,1.24]$ & 0.28 \\
\hline 4.1.6 Met+X vs X & 2 & 73 & $\mathrm{RR}(\mathrm{M}-\mathrm{H}, \mathrm{Random}, \quad 95 \% \mathrm{CI})$ & $0.35[0.12,0.96]$ & $0.04^{\mathrm{a}}$ \\
\hline
\end{tabular}

A study can be awarded a maximum of one star for each numbered item within the Selection and Outcome categories. A maximum of two stars can be given for Comparability

with 95\% confidence intervals (CIs). Heterogeneity measurement was performed by forest plots as well as calculating $\mathrm{I}^{2}$ (> $50 \%$ was considered extensive heterogeneity). A fixed-effects model was used to combine study results if heterogeneity was minimal; otherwise, the random-effects model was used. Potential publication bias was also examined qualitatively by funnel plots using RevMan software when the distribution of CI deviated significantly.

\section{Results}

\section{Study inclusion and basic characteristics}

The literature research initially resulted in 7098 potentially relevant publications (Medline: 1773, EMbase: 4179, Cochrane: 1146); after removing duplicates, the remaining 1123 records were screened via titles and abstracts. 91 full-text articles assessed for eligibility, after further assessment of eligibility criteria, 29 articles were excluded (23 studies did not mention certain observation indicators, and complete data were not available in the other 6 studies). Thus, a total of 62 articles were used in this study [9-70], involving 6571 patients; the studies varied from 46 RCTs, 16 cohort studies, that is, they were nonrandomized experimental studies (Fig. 1). The baseline characteristics of the included studies are presented in Table 1. The Methods and results of the metaanalysis are presented in Table 2.

\section{Quality assessment of the included studies}

Bias in the included studies was assessed by different tools. Figure 2 illustrates the risk of bias of the RCTs. Both selection and reporting bias were relatively low. The MINORS score of nonrandomized experimental studies is shown in the last column of Table 3.

\section{Meta-analysis}

Sixty-two eligible studies were included in the metaanalysis of metformin vs control. The intervention approach was slightly complicated between studies. Therefore, we analyzed the data in different subgroups. EMT and endometrium artery RI were considered-primary outcomes; the CPR and MR were secondary outcomes. Each outcome measurement is described in forest plots in Figs. 3, 4, 5 and 6. 


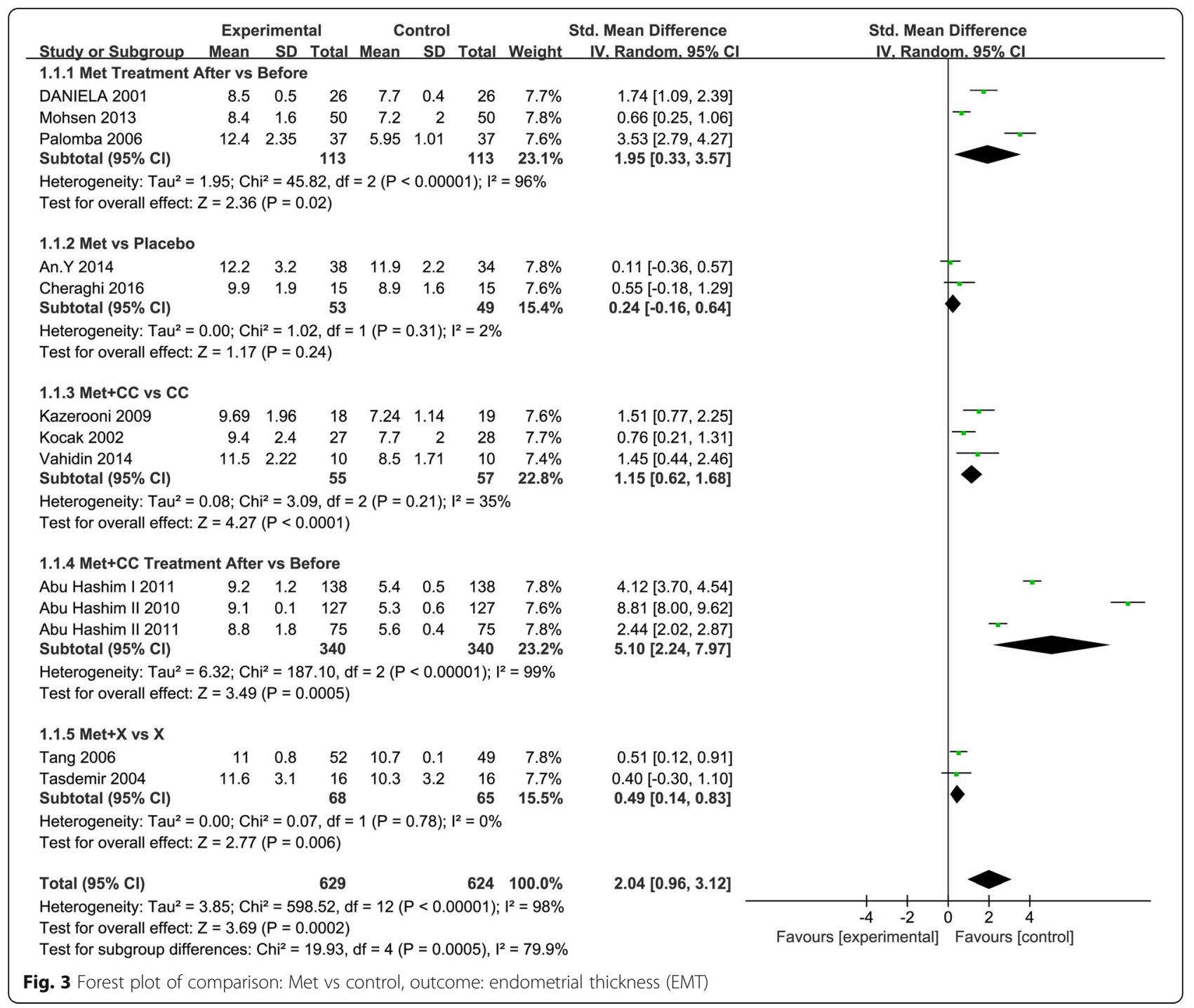

\section{Endometrial thickness (EMT)}

EMT was determined in thirteen studies. The randomeffects model showed that EMT in the metformin group was significantly thicker than that in the control group after treatment $(\mathrm{SMD}=2.04,95 \% \mathrm{CI}(0.96,3.12), P=$ 0.0002). (Fig. 3).

\section{Endometrium artery $\mathrm{RI}$}

The endometrium artery RI was reported in three studies. According to the random-effects model, the endometrium artery RI in the metformin group was significantly smaller than that in the control group after treatment (SMD = 2.83, 95\% CI: (- 5.06, - 0.59), $P=0.01)$. (Fig. 4).

\section{Clinical pregnancy rate (CPR)}

The CPR was described in Fifty-six studies. As revealed by the random-effects model, the clinical pregnancy rate in the metformin group was significantly different from that in the control group $(\mathrm{RR}=1.26,95 \% \mathrm{CI}: 1.11-1.43$, $P=0.0003$ ). (Fig. 5).

\section{Miscarriage rate (MR)}

Twenty-seven studies included the MR. The randomeffects model was applied, which indicated that the clinical pregnancy rate in the metformin group was significantly lower than that in the control group $(R R=0.73$, 95\% CI:0.58-0.91, $P=0.006$ ). (Fig. 6).

\section{Publication bias analysis}

In terms of publication bias estimation, as shown in Fig. 7, funnel plots indicated a relatively low likelihood of publication bias.

\section{Discussion}

In recent years, endometrial factors have been responsible for $1 / 3 \sim 2 / 3$ of pregnancies loss; however, the 


\begin{tabular}{|c|c|c|c|c|c|c|c|c|c|c|}
\hline \multirow[b]{2}{*}{ Study or Subgroup } & \multicolumn{3}{|c|}{ Experimental } & \multicolumn{3}{|c|}{ Control } & \multicolumn{2}{|c|}{ Std. Mean Difference } & \multirow{2}{*}{\multicolumn{2}{|c|}{$\begin{array}{l}\text { Std. Mean Difference } \\
\text { IV. Random. } 95 \% \mathrm{Cl}\end{array}$}} \\
\hline & Mean & SD & [otal & Mean & SD & Total & Weight & IV. Random. $95 \% \mathrm{Cl}$ & & \\
\hline \multicolumn{11}{|c|}{ 2.1.1 Met Treatment After vs Before } \\
\hline Mohsen 2013 & 0.83 & 1 & 50 & 0.85 & 0.75 & 50 & $25.7 \%$ & $-0.02[-0.41,0.37]$ & & T \\
\hline Palomba 2006 & 0.58 & 0.08 & 37 & 0.9 & 0.14 & 37 & $25.3 \%$ & $-2.78[-3.42,-2.13]$ & & \\
\hline Subtotal $(95 \% \mathrm{Cl})$ & & & 87 & & & 87 & $51.0 \%$ & $-1.39[-4.09,1.31]$ & & \\
\hline \multicolumn{11}{|c|}{$\begin{array}{l}\text { Heterogeneity: } \mathrm{Tau}^{2}=3.72 ; \mathrm{Chi}^{2}=50.90, \mathrm{df}=1(\mathrm{P}<0.00001) ; \mathrm{I}^{2}=98 \% \\
\text { Test for overall effect: } Z=1.01(\mathrm{P}=0.31)\end{array}$} \\
\hline \multicolumn{11}{|c|}{ 2.1.2 Met+CC Treatment After vs Before } \\
\hline $\begin{array}{l}\text { DANIELA } 2001 \\
\text { Subtotal }(95 \% \mathrm{Cl})\end{array}$ & 0.57 & 0.03 & $\begin{array}{l}26 \\
26\end{array}$ & 0.71 & 0.02 & $\begin{array}{l}26 \\
26\end{array}$ & $\begin{array}{l}24.1 \% \\
24.1 \%\end{array}$ & $\begin{array}{l}-5.41[-6.62,-4.20] \\
-5.41[-6.62,-4.20]\end{array}$ & & \\
\hline \multicolumn{11}{|c|}{ Heterogeneity: Not applicable } \\
\hline \multicolumn{11}{|c|}{ Test for overall effect: $Z=8.76(P<0.00001)$} \\
\hline \multicolumn{11}{|l|}{ 2.1.3 Met+CC vs CC } \\
\hline $\begin{array}{l}\text { DANIELA } 2001 \\
\text { Subtotal }(95 \% \mathrm{Cl})\end{array}$ & 0.57 & 0.03 & $\begin{array}{l}26 \\
26\end{array}$ & 0.67 & 0.03 & $\begin{array}{l}26 \\
26\end{array}$ & $\begin{array}{l}24.9 \% \\
24.9 \%\end{array}$ & $\begin{array}{l}-3.28[-4.14,-2.43] \\
-3.28[-4.14,-2.43]\end{array}$ & & \\
\hline \multirow{2}{*}{\multicolumn{11}{|c|}{ Heterogeneity: Not applicable }} \\
\hline \multicolumn{3}{|c|}{ Test for overall effect: $Z=7.55(P<0.00001)$} & & & & & & & & \\
\hline Total $(95 \% \mathrm{Cl})$ & & & 139 & & & 139 & $100.0 \%$ & $-2.83[-5.06,-0.59]$ & & \\
\hline \multicolumn{9}{|c|}{$\begin{array}{l}\text { Heterogeneity: } \mathrm{Tau}^{2}=5.03 ; \mathrm{Chi}^{2}=125.68, \mathrm{df}=3(\mathrm{P}<0.00001) ; \mathrm{I}^{2}=98 \% \\
\text { Test for overall effect: } Z=2.48(P=0.01) \\
\text { Test for subaroun differences: } \mathrm{Chi}^{2}=11.26 . \mathrm{df}=2(\mathrm{P}=0.004) . \mathrm{I}^{2}=82.2 \%\end{array}$} & $\begin{array}{ccc}-4 & -2 & 0 \\
\text { Favours [experimental] }\end{array}$ & $\begin{array}{cc}2 & 4 \\
\text { Favours [control] }\end{array}$ \\
\hline \multicolumn{11}{|c|}{ Fig. 4 Forest plot of comparison: Met vs control, outcome: endometrium artery RI } \\
\hline
\end{tabular}

underlying mechanisms related to ER remain unclear and need further investigation [71]. It is well established that ER insufficiency is associated with several gynecological diseases, such as hydrosalpinx, endometriosis, and uterine fibroids [72]. In addition, PCOS, a pathological condition with high serum androgen levels, can impede ER. Some studies report several ER-related markers, including integrin, MMP-9, TMP1, VEGF and LIF, are significantly decreased in the endometrium of PCOS patients during the window of implantation compared with normal controls [73]. Other studies have found that HOXA-10 and IGFBP-1, molecules associated with embryo development and endometrial decidualization, are downregulated in the endometrium of PCOS patients. It is considered that the mechanism of ER reduction in PCOS patients may be explained in three aspects. First, PCOS patients usually experience amenorrhea or oligomenorrhea, which causes a relatively low progesterone level. Second, the progesterone-lacking endometrium undergoes long-term exposure to estrogen, which leads to a continuous proliferative phase of the endometrium and may eventually impede ER establishment. Third, PCOS patients usually have complications with insulin resistance and hyperinsulinemia. Moreover, glucose metabolism is important for endometrial preparation for embryonic implantation, especially for endometrial decidualization. The transport of glucose from outside to inside the cell is mediated by a kind of special transporter, GLUT, which is responsible for glucose intake in many tissues under the influence of insulin, including the human endometrium. Expression of GLUTs in PCOS patients with insulin resistance is significantly decreased in the endometrium compared with women with obesity but without PCOS. Glucose metabolism disorders may exacerbate ER and lead to a series of alterations in the endometrium, including ER molecular marker expression and macro indicators. Consistently, it has been reported that the serum level of insulin directly alters expression of ER-related markers.

Metformin, as a sensitizer of insulin, has been used for PCOS patients with insulin resistance to facilitate fertility restoration [74]. The application of metformin can increase GLUTs expression in the endometrium of PCOS patients; however, its effects possible mechanisms on ER are remain poorly understood.

In 2019, Jun Zhai reported that metformin could improve ER by downregulating expression of miR-491-3p and miR-1910-3p, thereby increasing expression of HOXA10 and ITGB3, known as important endometrial receptive markers, in the endometrium of PCOS women [75-77]. The expression level of these microRNAs also increased with up-regulating metformin concentration, showing a clear dose-dependent manner. Second, other studies have suggested that metformin inhibited estradiol and progesterone-induced endometrial stroma by regulating the p38 MAPK signaling pathway and by changing the expression of various cytokines, such as MMP-2, MMP-9, ER and PGR, which are involved in the decidualization of stromal cells [78]. Decidualization refers to the conversion from endometrial stromal fibroblasts to specialized secretory decidual cell, which provide a nutrient and immune privileged matrix and are essential for embryo implantation and placental development [79]. Third, some studies have documented that 


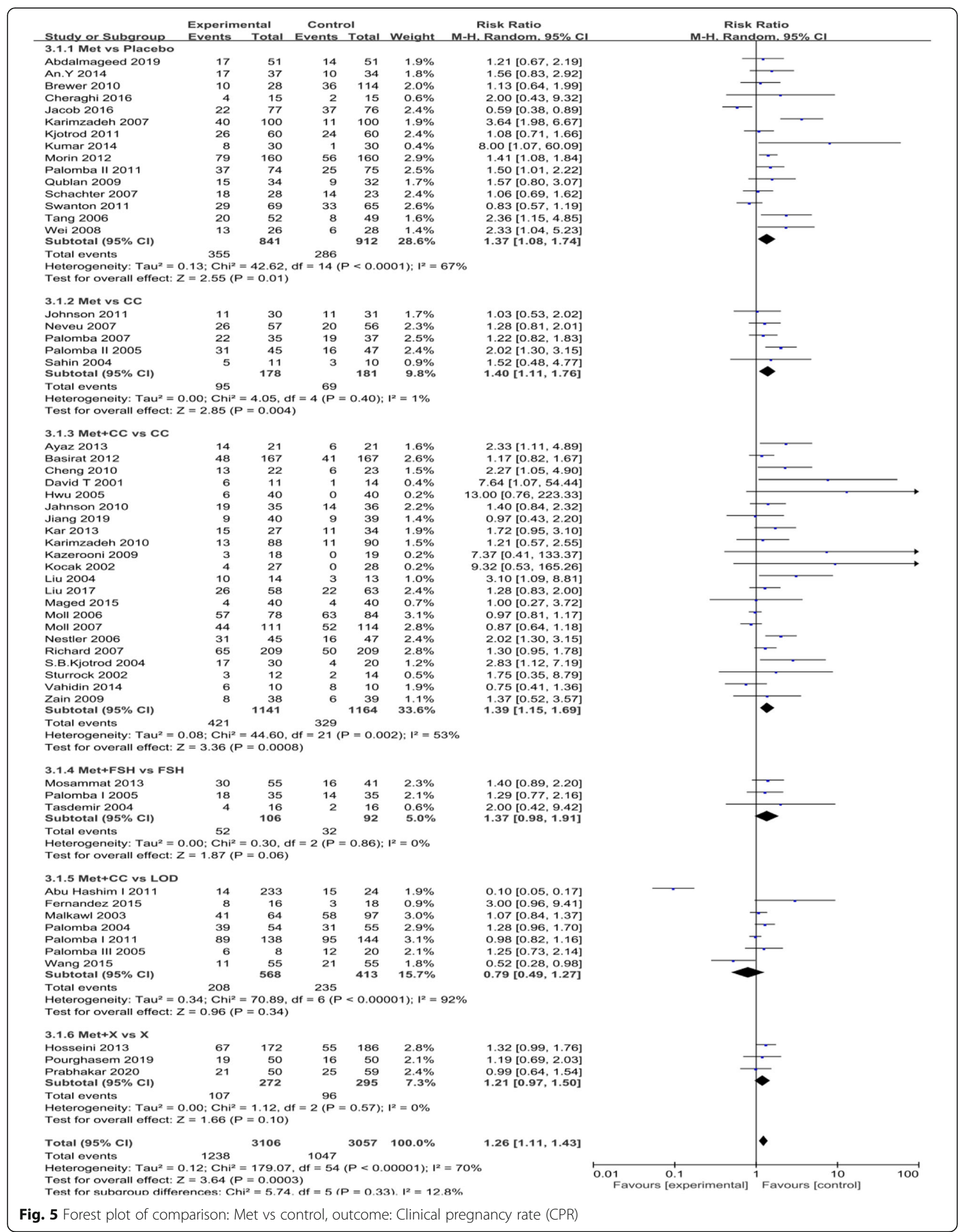




\begin{tabular}{|c|c|c|c|c|c|c|c|c|c|c|}
\hline \multirow{2}{*}{$\begin{array}{l}\text { Study or Subgroup } \\
\text { 4.1.1 Met vs Placebo }\end{array}$} & \multicolumn{2}{|c|}{ Experimental } & \multicolumn{2}{|c|}{ Control } & Weight & \multirow[t]{2}{*}{$\begin{array}{c}\text { Risk Ratio } \\
\text { M-H. Random, } 95 \% \mathrm{Cl}\end{array}$} & \multicolumn{4}{|c|}{$\begin{array}{c}\text { Risk Ratio } \\
\text { M-H. Random. } 95 \% \text { Cl }\end{array}$} \\
\hline & & & & & Weight & & \\
\hline Abdalmageed 2019 & 4 & 17 & 5 & 14 & $4.1 \%$ & $0.66[0.22,2.00]$ & & & - & \\
\hline Brewer 2010 & 2 & 10 & 11 & 36 & $2.9 \%$ & $0.65[0.17,2.48]$ & & & & \\
\hline Jahnson 2010 & 1 & 7 & 2 & 5 & $1.1 \%$ & $0.36[0.04,2.94]$ & & & & \\
\hline Morin 2012 & 12 & 79 & 10 & 56 & $8.6 \%$ & $0.85[0.40,1.83]$ & & & - & \\
\hline Palomba II 2011 & 5 & 26 & 5 & 24 & $4.1 \%$ & $0.92[0.30,2.80]$ & & & & \\
\hline Qublan 2009 & 4 & 15 & 3 & 9 & $3.3 \%$ & $0.80[0.23,2.79]$ & & & & \\
\hline Schachter 2007 & 7 & 18 & 7 & 14 & $8.3 \%$ & $0.78[0.36,1.70]$ & & & & \\
\hline Wei 2008 & 3 & 13 & 1 & 6 & $1.2 \%$ & $1.38[0.18,10.71]$ & & & & \\
\hline Subtotal $(95 \% \mathrm{Cl})$ & & 185 & & 164 & $33.7 \%$ & $0.78[0.53,1.15]$ & & & & \\
\hline Total events & 38 & & 44 & & & & & & & \\
\hline \multirow{2}{*}{\multicolumn{11}{|c|}{$\begin{array}{l}\text { Heterogeneity: } \text { Tau }^{2}=0.00 ; \mathrm{Chi}^{2}=1.12, \mathrm{df}=7(P=0.99) ; 1^{2}=0 \% \\
\text { Test for overall effect: } Z=1.24(P=0.22)\end{array}$}} \\
\hline & & & & & & & & & & \\
\hline 4.1.2 Met vs CC & & & & & & & & & & \\
\hline Baran 2010 & 1 & 11 & 1 & 11 & $0.7 \%$ & $1.00[0.07,14.05]$ & & & & \\
\hline Nestler 2006 & 3 & 31 & 6 & 16 & $3.3 \%$ & $0.26[0.07,0.90]$ & & & & \\
\hline Neveu 2007 & 5 & 26 & 3 & 20 & $3.0 \%$ & $1.28[0.35,4.74]$ & & & & \\
\hline Palomba 2007 & 4 & 22 & 5 & 19 & $3.8 \%$ & $0.69[0.22,2.21]$ & & & & \\
\hline Palomba II 2005 & 3 & 31 & 6 & 16 & $3.3 \%$ & $0.26[0.07,0.90]$ & & & & \\
\hline Subtotal $(95 \% \mathrm{Cl})$ & & 121 & & 82 & $14.0 \%$ & $0.51[0.26,0.99]$ & & & & \\
\hline Total events & 16 & & 21 & & & & & & & \\
\hline Heterogeneity: $\operatorname{Tau}^{2}=$ & $.09 ; \mathrm{Chi}^{2}=$ & $=4.71, \mathrm{df}$ & df $=4(P=$ & $0.32) ;$ & $I^{2}=15 \%$ & & & & & \\
\hline Test for overall effect: & $z=1.99(P$ & $=0.05)$ & & & & & & & & \\
\hline 4.1.3 Met+CC vs CC & & & & & & & & & & \\
\hline Jiang 2019 & 3 & 9 & 7 & 9 & $5.2 \%$ & $0.43[0.16,1.15]$ & & & & \\
\hline Liu 2017 & 7 & 26 & 3 & 33 & $3.2 \%$ & $2.96[0.85,10.35]$ & & & & \\
\hline Moll 2006 & 13 & 57 & 12 & 63 & $10.4 \%$ & $1.20[0.60,2.41]$ & & & & \\
\hline S.B.Kjotrod 2004 & 3 & 13 & 8 & 17 & $4.1 \%$ & $0.49[0.16,1.49]$ & & & - & \\
\hline Subtotal $(95 \% \mathrm{Cl})$ & & 105 & & 122 & $23.0 \%$ & $0.91[0.41,1.98]$ & & & & \\
\hline Total events & 26 & & 30 & & & & & & & \\
\hline Heterogeneity: $\mathrm{Tau}^{2}=$ & $0.38 ; \mathrm{Chi}^{2}=$ & $=7.54, \mathrm{df}$ & if $=3(P=$ & 0.06 ); & $I^{2}=60 \%$ & & & & & \\
\hline Test for overall effect: & $z=0.25(P$ & $=0.80)$ & & & & & & & & \\
\hline 4.1.4 Met+FSH vs FS & & & & & & & & & & \\
\hline Mosammat 2013 & 1 & 18 & 0 & 14 & $0.5 \%$ & $2.37[0.10,54.08]$ & & & & \\
\hline Palomba I 2005 & 3 & 30 & 3 & 16 & $2.3 \%$ & $0.53[0.12,2.34]$ & & & & \\
\hline Subtotal $(95 \% \mathrm{Cl})$ & & 48 & & 30 & $2.8 \%$ & $0.70[0.18,2.67]$ & & & & \\
\hline Total events & 4 & & 3 & & & & & & & \\
\hline Heterogeneity: Tau $^{2}=$ & $0.00 ; \mathrm{Chi}^{2}=$ & $=0.73, \mathrm{df}$ & If $=1(P=$ & 0.39); & $1^{2}=0 \%$ & & & & & \\
\hline Test for overall effect: & $z=0.52(P$ & $=0.60)$ & & & & & & & & \\
\hline 4.1.5 Met+CC vs LOD & & & & & & & & & & \\
\hline Abu Hashim I 2011 & 2 & 14 & 2 & 15 & $1.5 \%$ & $1.07[0.17,6.61]$ & & & & \\
\hline Malkawl 2003 & 6 & 39 & 9 & 31 & $6.0 \%$ & $0.53[0.21,1.33]$ & & & - & \\
\hline Palomba 2004 & 3 & 41 & 6 & 58 & $2.9 \%$ & $0.71[0.19,2.67]$ & & & & \\
\hline Palomba I 2011 & 8 & 89 & 9 & 95 & $6.2 \%$ & $0.95[0.38,2.35]$ & & & & \\
\hline Palomba III 2005 & 2 & 6 & 5 & 12 & $2.9 \%$ & $0.80[0.21,2.98]$ & & & & \\
\hline Wang 2015 & 2 & 11 & 4 & 21 & $2.2 \%$ & $0.95[0.21,4.42]$ & & & & \\
\hline Subtotal $(95 \% \mathrm{Cl})$ & & 200 & & 232 & $21.7 \%$ & $0.77[0.47,1.24]$ & & & & \\
\hline Total events & 23 & & 35 & & & & & & & \\
\hline Heterogeneity: $\mathrm{Tau}^{2}=$ & 0.00; $\mathrm{Chi}^{2}=$ & $=1.06, \mathrm{df}$ & If $=5(P=$ & $0.96) ;$ & $1^{2}=0 \%$ & & & & & \\
\hline Test for overall effect: & $Z=1.08(P$ & $=0.28)$ & & & & & & & & \\
\hline 4.1.6 Met+X vs $X$ & & & & & & & & & & \\
\hline Abu Hashim I 2010 & 4 & 22 & 3 & 5 & $3.9 \%$ & $0.30[0.10,0.95]$ & & & & \\
\hline Prabhakar 2020 & 1 & 21 & 2 & 25 & $0.9 \%$ & $0.60[0.06,6.11]$ & & & & \\
\hline Subtotal $(95 \% \mathrm{Cl})$ & & 43 & & 30 & $4.8 \%$ & $0.35[0.12,0.96]$ & & & & \\
\hline Total events & 5 & & 5 & & & & & & & \\
\hline Heterogeneity: $\mathrm{Tau}^{2}=$ & $0.00 ; \mathrm{Chi}^{2}=$ & $=0.30, \mathrm{df}$ & $f=1(P=$ & $0.58) ;$ & $I^{2}=0 \%$ & & & & & \\
\hline Test for overall effect: & $z=2.04(P$ & $=0.04)$ & & & & & & & & \\
\hline Total $(95 \% \mathrm{Cl})$ & & 702 & & 660 & $100.0 \%$ & $0.73[0.58,0.91]$ & & $\bullet$ & & \\
\hline Total events & 112 & & 138 & & & & & & & \\
\hline Heterogeneity: Tau $^{2}=$ & 0.00; $\mathrm{Chi}^{2}=$ & $=19.93, \mathrm{c}$ & $\mathrm{df}=26(\mathrm{P}$ & $=0.79$ & $9) ; 1^{2}=0 \%$ & & 0.01 & $\begin{array}{ll}0.1 & 1\end{array}$ & & 100 \\
\hline Test for overall effect: & $Z=2.75(P$ & $=0.006)$ & & & & & & vours [experimental] & Favours [control] & \\
\hline Test for subaroub diffe & rences: $\mathrm{Ch}$ & $i^{2}=3.56$ & $d f=5(P$ & $=0.61$ & 1). $\left.\right|^{2}=0 \%$ & & & & & \\
\hline orest plot of compa & rison: Me & vs con & trol, ou & m & :Miscar & Corato (A & & & & \\
\hline
\end{tabular}


(A)

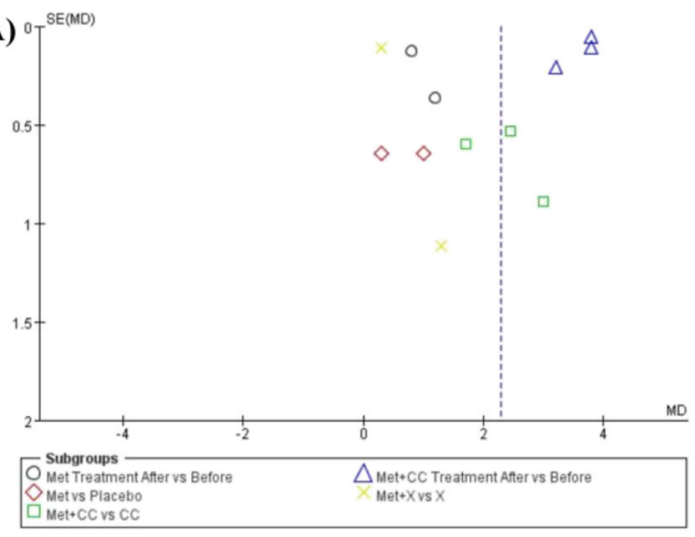

(C) ${ }^{\circ}$

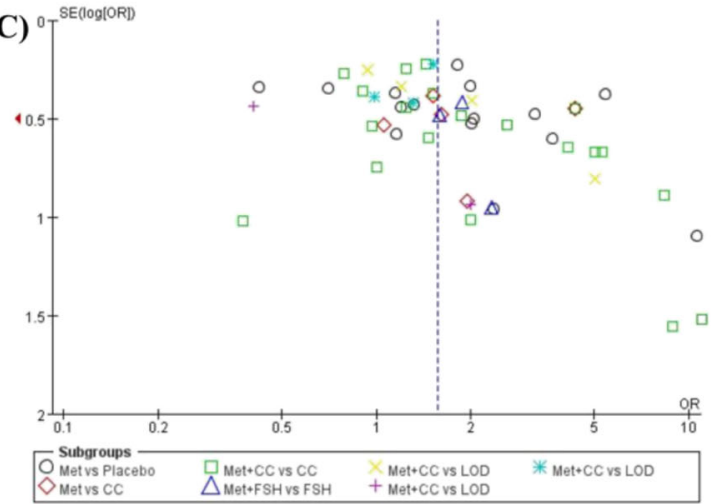

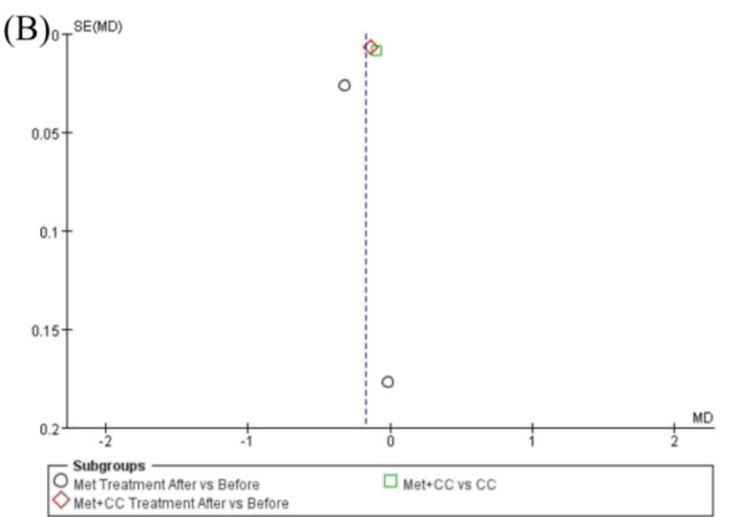

(D)

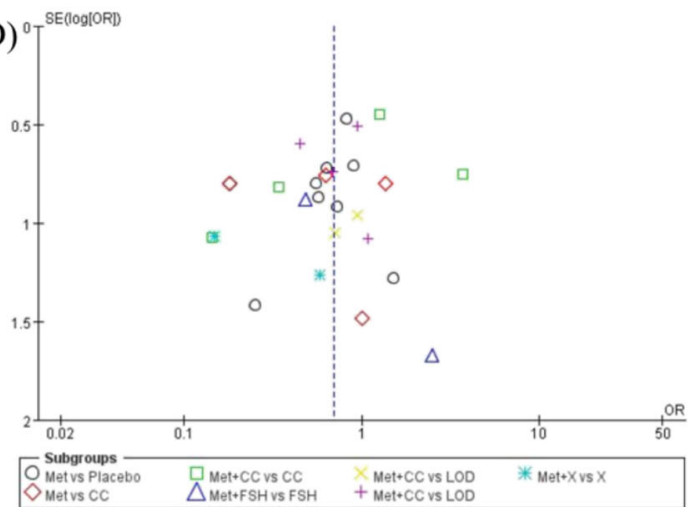

(A)Endometrial Thickness; (B)Endometrium Artery RI; (C)Clinical pregnancy rate; (D)Miscarriage

rate.

Fig. 7 Funnel plot of comparison: Met vs control

endometrial GLUT4 protein and mRNA expression were significantly increased after Met treatment in PCOS patients. Hyperglycemia and hyperinsulinemia may reduce GLUT4 expression in adipocytes, and Met can reverse this process and promote GLUT4 translocation [80]. GLUT4 express decreased in the endometrium may reduce glucose transmembrane transport, affect cell glucose utilization, and lead to endometrial embolism; subsequently, embryo implantation failure and miscarriage occur. However, it was also observed that expression of GLUT4 protein and mRNA in the endometrium of PCOS patients was increased after Met treatment. Therefore, Met may upregulate GLUT4 expression in the endometrium of PCOS patients and improve ER.

There is an assumption that Met can promote endometrium decidualization by increasing the expression of GLUT4 in the endometrium or providing enough energy for thickening of endometrium. Move over, it is also reported that it takes a long time to remodel and develop blood vessels in the placenta since the embryo implantation. At this stage, the embryo is exposed to a hypoxic environment. Changes in oxygen pressure cause multiple functional responses, such as adaptive responses to reduced oxygen concentrations or alternative metabolic pathways to provide oxygen energy substrates [81]. Therefore, we suggested that when the endometrial artery RI during embryo implantation is lower the blood supply for endometrium is better; that is, the RI of the endometrial artery is lower and more conducive to the remodeling and regeneration of blood vessels during embryo implantation. As mentioned above, treatment of PCOS with metformin can reduce the RI of the endometrial artery, and it is likely to provide a good environment for embryo implantation.

In this meta-analysis, the primary outcomes were EMT and endometrial artery RI. The main methods for evaluating ER are ultrasound, endometrial biopsy, endometrial fluid aspiration and hysteroscopy [82]. The application of ultrasound to evaluate ER can be divided into four aspects: EMT, endometrial volume, endometrial pattern and endometrial blood flow [83]. Currently, It is generally believed that EMT is the most common ER index. Some studies suggest a positive correlation between EMT and pregnancy rate [84-86], but there are 
also some reports no association [87]. Although the evaluation of ER in endometrial blood perfusion are constantly enriched, the most commonly used indicators are the PI and RI of the endometrial artery [88]. Many studies believe that the PI and RI of the endometrial artery can be used as effective indicators of ER $[89,90]$. However, some suggest that measurement of the ER index of the uterine spiral artery or endometrial artery cannot reliably predict the prognosis of IVF [91-93].

This systemic review and meta-analysis summarize studies investigating the effect of metformin on ER of PCOS patients. It is still unclear how the protective effects of Met in endometrial receptivity operates, and further research is needed. Moreover, due to the high heterogeneity of the included data and the large merger bias, the results need to be treated with caution.

\section{Limitations}

This meta-analysis had several limitations that should be taken into consideration when interpreting the conclusions. First, this meta-analysis summarized a total of 46 RCTs, 16 cohort studies, but the sample sizes were relatively small. Second, a poorly randomized design included studies, and the complex interventions made more biased in the study mergers. The results obtained were widely heterogeneous and considered to be the main limiting factor. According to the above limitations, caution should be used when evaluating the results of this meta-analysis.

\section{Implications}

The following issues should be considered in future study design. First, as the time to measure EMT may have different effects on the study results, the time to measure EMT or ER in all patients should be the same period. Second, the current study involved few evaluation indicators for ER and Endometrium artery RI. Future research should add up more evaluation markers for ER.

\section{Conclusion}

Overall, this systematic review and meta-analysis suggests that the effect of metformin for improving endometrial receptivity in women with PCOS is weak but meaningful. Notably, the sample size of the studies was not large, and the evidence was high quality albeit insufficient. Therefore, large-scale and multiple centers RCTs with rigorous methodological quality are needed to clarify the role of metformin in ER. Further research is needed to explore the long-term efficacy and the mechanisms of the intervention.

\section{Abbreviations}

Met: Metformin; PCOS: Polycyclic ovary syndrome; ER: Endometrial receptivity; EMT: Endometrial thickness; RI: Resistance index; CPR: Clinical pregnancy rate; MR: Miscarriage rate; PI: Pulsatility index; RCT: Randomized controlled trials; ARTs: Assisted reproductive technologies; IVF: In vitro fertilization; ET: Embryo transfer

\section{Acknowledgements}

The authors thank Ming Liao for nice help on methodology.

\section{Authors' contributions}

YLF and WHB performed the literature research, collated the data and drafted the initial manuscript. HWY and BY reviewed the full text of relevant articles and checked the data. YHY and QAP provided statistical analysis method. YLF and HWY performed the meta-analysis and interpreted the results. YYH revised the manuscript critically for important intellectual content. YYH and QAP provided professional suggestions and resolved any problems and disagreements. All authors read and approved the final manuscript.

\section{Funding}

This study was supported by grants from the National Natural Science Foundation of China (NO. 81871172), the Natural Science Foundation of Guangxi Zhuang Autonomous Region (NO. 2018GXNSFDA050017 and NO. 2019GXNSFFA245013), the Guangxi Medical University Training Program for Distinguished Young Scholars and Guangxi Scholarship Fund of Guangxi

Education Department of China.

Availability of data and materials

All data are fully available without restriction.

\section{Declarations}

Ethics approval and consent to participate Not applicable.

\section{Consent for publication}

Written informed consent for publication was obtained from all participants.

\section{Competing interests}

There is no conflict of interest.

\section{Author details}

${ }^{1}$ Reproductive Medical Center, the First Affiliated Hospital of Guangxi Medical University, Nanning, China. ${ }^{2}$ Reproductive Medical Center, Qinzhou Women and Children Hospital, Qinzhou, China.

Received: 4 February 2021 Accepted: 28 May 2021

Published online: 14 June 2021

\section{References}

1. Salat-Baroux J, Cornet D, Alvarez S, Antoine JM, de Brux J, Firmin C, et al. Evaluation criteria of the condition of the endometrium in relation to the presence of ovocytes. Rev Fr Gynecol et obstet. 1988;83(10):603-6.

2. Lessey BA, Young SL. What exactly is endometrial receptivity? Fertil Steril. 2019;111(4):611-7. https://doi.org/10.1016/j.fertnstert.2019.02.009.

3. Valdes $C T$, Schutt A, Simon C. Implantation failure of endometrial origin: it is not pathology, but our failure to synchronize the developing embryo with a receptive endometrium. Fertil Steril. 2017;108(1):15-8. https://doi.org/10.101 6/j.fertnstert.2017.05.033.

4. Edwards RG. Human implantation: the last barrier in assisted reproduction technologies?. Reprod Biomed Online. 2007, 14 Spec No 1:5-22.

5. Miravet-Valenciano JA, Rincon-Bertolin A, Vilella F, Simon C. Understanding and improving endometrial receptivity. Curr Opin Obstet Gynecol. 2015: 27(3):187-92. https://doi.org/10.1097/GCO.0000000000000173.

6. Smith MB, Paulson RJ. Endometrial preparation for third-party parenting and cryopreserved embryo transfer. Fertil Steril. 2019;111(4):641-9. https://doi. org/10.1016/j.fertnstert.2019.02.010.

7. Costello MF, Garad RM, Hart R, et al. A Review of Second- and Third-line Infertility Treatments and Supporting Evidence in Women with Polycystic Ovary Syndrome. Med sci (Basel). 2019;7:7.

8. Sharpe A, Morley LC, Tang T, et al. Metformin for ovulation induction (excluding gonadotrophins) in women with polycystic ovary syndrome. Cochrane Database Syst Rev. 2019;12(12):Cd013505. 
9. Abdalmageed OS, Farghaly TA, Abdelaleem AA, et al. Impact of Metformin on IVF Outcomes in Overweight and Obese Women With Polycystic Ovary Syndrome: A Randomized Double-Blind Controlled Trial. Reprod Sci. 2019; 26(10):1336-42.

10. Abu Hashim H, Anwar K, El-Fatah RA. N-acetyl cysteine plus clomiphene citrate versus metformin and clomiphene citrate in treatment of clomiphene-resistant polycystic ovary syndrome: a randomized controlled trial. J Womens Health. 2010;19(11):2043-8.

11. Abu Hashim H, El Lakany N, Sherief L. Combined metformin and clomiphene citrate versus laparoscopic ovarian diathermy for ovulation induction in clomiphene-resistant women with polycystic ovary syndrome: a randomized controlled trial. J Obstet Gynaecol Res. 2011;37(3):169-77. https://doi.org/10.1111/j.1447-0756.2010.01383.x.

12. Abu Hashim H, Shokeir T, Badawy A. Letrozole versus combined metformin and clomiphene citrate for ovulation induction in clomiphene-resistant women with polycystic ovary syndrome: a randomized controlled trial. Fertil Steril. 2010;94(4):1405-9. https://doi.org/10.1016/j.fertnstert.2009.07.985.

13. Abu Hashim H, Wafa A, El Rakhawy M. Combined metformin and clomiphene citrate versus highly purified FSH for ovulation induction in clomiphene-resistant PCOS women: a randomised controlled trial. Gynecol Endocrinol. 2011;27(3):190-6. https://doi.org/10.3109/09513590.2010.488771.

14. An Y, Sun Z, Zhang Y, Liu B, Guan Y, Lu M. The use of berberine for women with polycystic ovary syndrome undergoing IVF treatment. Clin Endocrinol. 2014;80(3):425-31. https://doi.org/10.1111/cen.12294.

15. Ayaz A, Alwan Y, Faroog MU. Metformin-clomiphene citrate vs. clomiphene citrate alone: polycystic ovarian syndrome. J Human Reprod Sci. 2013;6(1): 15-8. https://doi.org/10.4103/0974-1208.112372.

16. Baran S, Api M, Goksedef BP, et al. Comparison of metformin and clomiphene citrate therapy for induction of ovulation in the polycystic ovary syndrome. Arch Gynecol Obstet. 2010;282(4):439-43. https://doi.org/1 0.1007/s00404-010-1497-y.

17. Basirat Z, Kashifard M, Amiri MG. Enhanced ovarian folliclular development by metformin does not correlate with pregnancy rate: a randomized trial. Int J Fertil Steril. 2012;6(1):31-6.

18. Begum MR, Akhter S, Ehsan M, Begum MS, Khan F. Pretreatment and coadministration of oral anti-diabetic agent with clomiphene citrate or rFSH for ovulation induction in clomiphene-citrate-resistant polycystic ovary syndrome. J Obstet Gynaecol Res. 2013;39(5):966-73. https://doi.org/1 0.1111/j.1447-0756.2012.02072.x.

19. Brewer C, Acharya S, Thake F, et al. Effect of metformin taken in the 'fresh' in vitro fertilization/intracytoplasmic sperm injection cycle upon subsequent frozen embryo replacement in women with polycystic ovary syndrome. Hum Fertil (Cambridge). 2010;13(3):134-42.

20. Cheng J, LV J, Li CY, Xue Y, Huang Z, Zheng W. Clinical outcomes of ovulation induction with metformin, clomiphene citrate and human menopausal gonadotrophin in polycystic ovary syndrome. J Int Med Res. 2010;38(4):1250-8. https://doi.org/10.1177/147323001003800406.

21. Cheraghi E, Mehranjani MS, Shariatzadeh MA, Esfahani MHN, Ebrahimi Z. Nacetylcysteine improves oocyte and embryo quality in polycystic ovary syndrome patients undergoing intracytoplasmic sperm injection: an alternative to metformin. Reprod Fertil Dev. 2016;28(6):723-31. https://doi. org/10.1071/RD14182.

22. Fernandez H, Cedrin-Durnerin I, Gallot V, Rongieres C, Watrelot A, MayengaMankezi JM, et al. Using an ovarian drilling by hydrolaparoscopy or recombinant follicle stimulating hormone plus metformin to treat polycystic ovary syndrome: why a randomized controlled trial fail? J Gynecol Obstet Biol Reprod (Paris). 2015;44(8):692-8. https://doi.org/10.1016/j.jgyn.2014.10.01 6.

23. Hosseini MA, Alleyassin A, Sarvi F, Safdarian L, Kokab A, Fanisalek M. Metformin treatment in different phenotypes of polycystic ovary syndrome. Arch Gynecol Obstet. 2013;288(5):1131-6. https://doi.org/10.1007/s00404013-2800-5.

24. Hwu YM, Lin SY, Huang WY, Lin MH, Lee RKK. Ultra-short metformin pretreatment for clomiphene citrate-resistant polycystic ovary syndrome. Int J Gynaecol Obstet. 2005;90(1):39-43. https://doi.org/10.1016/j.ijgo.2005.04. 004.

25. Jacob SL, Brewer C, Tang T, et al. A short course of metformin does not reduce OHSS in a GnRH antagonist cycle for women with PCOS undergoing IVF: a randomised placebo-controlled trial. Hum Reprod. 2016; 31(12):2756-64.
26. Jakubowicz DJ, Seppälä M, Jakubowicz S, Rodriguez-Armas O, RivasSantiago A, Koistinen $\mathrm{H}$, et al. Insulin reduction with metformin increases luteal phase serum glycodelin and insulin-like growth factor-binding protein 1 concentrations and enhances uterine vascularity and blood flow in the polycystic ovary syndrome. J Clin Endocrinol Metab. 2001;86(3):1126-33. https://doi.org/10.1210/jcem.86.3.7295.

27. Jiang J, Gao S, Zhang Y. Therapeutic effects of dimethyldiguanide combined with clomifene citrate in the treatment of polycystic ovary syndrome. Rev Assoc Med Bras (1992). 2019;65(9):1144-50.

28. Johnson NP, Bontekoe S, Stewart AW. Analysis of factors predicting success of metformin and clomiphene treatment for women with infertility owing to PCOS-related ovulation dysfunction in a randomised controlled trial. Aust N Z J Obstet Gynaecol. 2011;51(3):252-6. https://doi.org/10.1111/j.1479-82 8X.2011.01295.x.

29. Johnson NP, Stewart AW, Falkiner J, et al. PCOSMIC: a multi-centre randomized trial in women with PolyCystic Ovary Syndrome evaluating Metformin for Infertility with Clomiphene. Human Reprod. 2010;25(7):167583.

30. Kar S. Clomiphene citrate, metformin or the combination of both, as first line ovulation induction drug in polycystic ovarian syndrome: a randomised controlled trial. Fertil Steril. 2013;100(3):S359-S60.

31. Karimzadeh MA, Javedani M. An assessment of lifestyle modification versus medical treatment with clomiphene citrate, metformin, and clomiphene citrate-metformin in patients with polycystic ovary syndrome. Fertil Steril. 2010;94(1):216-20. https://doi.org/10.1016/j.fertnstert.2009.02.078.

32. Kazerooni T, Ghaffarpasand F, Kazerooni Y, Kazerooni M, Setoodeh S. Shortterm metformin treatment for clomiphene citrate-resistant women with polycystic ovary syndrome. Int J Gynaecol Obstet. 2009;107(1):50-3. https:// doi.org/10.1016/j.ijgo.2009.04.022.

33. Kjøtrød SB, Carlsen SM, Rasmussen PE, et al. Use of metformin before and during assisted reproductive technology in non-obese young infertile women with polycystic ovary syndrome: a prospective, randomized, double-blind, multi-centre study. Hum Reprod. 2011;26(8):2045-53.

34. Kjøtrød SB, von Düring V, Carlsen SM. Metformin treatment before IVF/ICSI in women with polycystic ovary syndrome; a prospective, randomized, double blind study. Hum Reprod. 2004;19(6):1315-22.

35. Kocak M, Caliskan E, Simsir C, Haberal A. Metformin therapy improves ovulatory rates, cervical scores, and pregnancy rates in clomiphene citrateresistant women with polycystic ovary syndrome. Fertil Steril. 2002;77(1): 101-6. https://doi.org/10.1016/S0015-0282(01)02941-7.

36. Kumar P, Arora S. Orlistat in polycystic ovarian syndrome reduces weight with improvement in lipid profile and pregnancy rates. J Hum Reprod Sci. 2014;7(4):255-61. https://doi.org/10.4103/0974-1208.147492.

37. Legro RS, Barnhart HX, Schlaff WD, Carr BR, Diamond MP, Carson SA, et al. Clomiphene, metformin, or both for infertility in the polycystic ovary syndrome. N Engl J Med. 2007;356(6):551-66. https://doi.org/10.1056/ NEJMoa063971.

38. Liu C, Feng G, Huang W, Wang Q, Yang S, Tan J, et al. Comparison of clomiphene citrate and letrozole for ovulation induction in women with polycystic ovary syndrome: a prospective randomized trial. Gynecol Endocrinol. 2017;33(11):872-6. https://doi.org/10.1080/09513590.2017.13321 74

39. Liu ZA, Xue YM, Chen LX, Cai Q, Chen H, Zhang J, et al. Clinical study on treating insulin resistance and promoting ovulation in polycystic ovary syndrome. Zhonghua Fu Chan Ke Za Zhi. 2004;39(9):586-90.

40. Maged AM, Elsawah H, Abdelhafez A, Bakry A, Mostafa WAl. The adjuvant effect of metformin and $\mathrm{N}$-acetylcysteine to clomiphene citrate in induction of ovulation in patients with polycystic ovary syndrome. Gynecol Endocrinol. 2015;31(8):635-8. https://doi.org/10.3109/09513590.2015.10372 69.

41. Malkawi HY, Qublan HS, Hamaideh AH. Medical vs. surgical treatment for clomiphene citrate-resistant women with polycystic ovary syndrome. J Obstet Gynaecol. 2003;23(3):289-93. https://doi.org/10.1080/0144361031 0000100123.

42. Karimzadeh MA, Eftekhar M, Taheripanah $\mathrm{R}$. The effect of administration of metformin on lipid profile changes and insulin resistance in patients with polycystic ovary syndrome. Middle East Fertil Soc J. 2007;12(3):174.

43. Mohsen IA, Elkattan E, Nabil H, Khattab S. Effect of metformin treatment on endometrial vascular indices in anovulatory obese/overweight women with polycystic ovarian syndrome using three-dimensional power doppler 
ultrasonography. J Clin Ultrasound. 2013;41(5):275-82. https://doi.org/10.1 002/jcu.22006

44. Moll E, Bossuyt PM, Korevaar JC, et al. Effect of clomifene citrate plus metformin and clomifene citrate plus placebo on induction of ovulation in women with newly diagnosed polycystic ovary syndrome: randomised double blind clinical trial. BMJ. 2006;332(7556):1485.

45. Moll E, Korevaar JC, Bossuyt PM, et al. Does adding metformin to clomifene citrate lead to higher pregnancy rates in a subset of women with polycystic ovary syndrome? Hum Reprod. 2008;23(8):1830-4.

46. Morin-Papunen L, Rantala AS, Unkila-Kallio L, Tiitinen A, Hippeläinen M, Perheentupa A, et al. Metformin improves pregnancy and live-birth rates in women with polycystic ovary syndrome (PCOS): a multicenter, double-blind, placebo-controlled randomized trial. J Clin Endocrinol Metab. 2012;97(5): 1492-500. https://doi.org/10.1210/jc.2011-3061.

47. Nestler JE. Is metformin or clomiphene citrate more effective for ovulation induction in polycystic ovary syndrome? Nat Clin Pract Endocrinol Metab. 2006;2(3):128-9. https://doi.org/10.1038/ncpendmet0113.

48. Neveu N, Granger L, St-Michel P, et al. Comparison of clomiphene citrate, metformin, or the combination of both for first-line ovulation induction and achievement of pregnancy in 154 women with polycystic ovary syndrome. Fertil Steril. 2007:87(1):113-20. https://doi.org/10.1016/j.fertnstert.2006.05.069.

49. Ota H, Goto T, Yoshioka T, et al. Successful pregnancies treated with pioglitazone in infertile patients with polycystic ovary syndrome. Fertil Steril. 2008;90(3):709-13. https://doi.org/10.1016/j.fertnstert.2007.01.117.

50. Palomba S, Falbo A, Battista L, et al. Laparoscopic ovarian diathermy vs clomiphene citrate plus metformin as second-line strategy for infertile anovulatory patients with polycystic ovary syndrome: a randomized controlled trial. Am J Obstet Gynecol. 2010;202(6):577.e1-8.

51. Palomba S, Falbo A, Carrillo L, et al. Metformin reduces risk of ovarian hyperstimulation syndrome in patients with polycystic ovary syndrome during gonadotropin-stimulated in vitro fertilization cycles: a randomized, controlled trial. Fertil Steril. 2011;96(6):1384-90.e4.

52. Palomba S, Falbo A, Orio F Jr, et al. A randomized controlled trial evaluating metformin pre-treatment and co-administration in non-obese insulinresistant women with polycystic ovary syndrome treated with controlled ovarian stimulation plus timed intercourse or intrauterine insemination. Hum reprod. 2005;20(10):2879-86.

53. Palomba S, Orio F Jr, Falbo A, Manguso F, Russo T, Cascella T, et al. Prospective parallel randomized, double-blind, double-dummy controlled clinical trial comparing clomiphene citrate and metformin as the first-line treatment for ovulation induction in nonobese anovulatory women with polycystic ovary syndrome. J Clin Endocrinol Metab. 2005;90(7):4068-74. https://doi.org/10.1210/jc.2005-0110.

54. Palomba S, Orio F Jr, Falbo A, et al. Metformin administration and laparoscopic ovarian drilling improve ovarian response to clomiphene citrate (CC) in oligoanovulatory CC-resistant women with polycystic ovary syndrome. Clin Endocrinol. 2005;63(6):631-5. https://doi.org/10.1111/j.1365-2265.2005.02392.x.

55. Palomba S, Orio F Jr, Falbo A, Russo T, Tolino A, Zullo F. Clomiphene citrate versus metformin as first-line approach for the treatment of anovulation in infertile patients with polycystic ovary syndrome. J Clin Endocrinol Metab. 2007;92(9):3498-503. https://doi.org/10.1210/jc.2007-1009.

56. Palomba S, Orio F Jr, Nardo LG, Falbo A, Russo T, Corea D, et al. Metformin administration versus laparoscopic ovarian diathermy in clomiphene citrateresistant women with polycystic ovary syndrome: a prospective parallel randomized double-blind placebo-controlled trial. J Clin Endocrinol Metab. 2004;89(10):4801-9. https://doi.org/10.1210/jc.2004-0689.

57. Palomba S, Russo T, Orio F Jr, et al. Uterine effects of metformin administration in anovulatory women with polycystic ovary syndrome. Hum Reprod. 2006; 21(2):457-65.

58. Pourghasem S, Bazarganipour F, Taghavi SA, Kutenaee MA. The effectiveness of inositol and metformin on infertile polycystic ovary syndrome women with resistant to letrozole. Arch Gynecol Obstet. 2019; 299(4):1193-9. https://doi.org/10.1007/s00404-019-05064-5.

59. Prabhakar P, Mahey R, Gupta M et al. Impact of myoinositol with metformin and myoinositol alone in infertile PCOS women undergoing ovulation induction cycles - randomized controlled trial. Gynecol Endocrinol. 2021;37(4):332-6. https:/ doi.org/10.1080/09513590.2020.1810657. Epub 2020 Sep 18. PMID: 32945218.

60. Qublan HS, Al-Khaderei S, Abu-Salem AN, et al. Metformin in the treatment of clomiphene citrate-resistant women with polycystic ovary syndrome undergoing in vitro fertilisation treatment: a randomised controlled trial. J Obstet Gynaecol. 2009;29(7):651-5. https://doi.org/10.1080/01443610903147576.
61. Sahin $Y$, Yirmibeş $U$, Keleştimur F, et al. The effects of metformin on insulin resistance, clomiphene-induced ovulation and pregnancy rates in women with polycystic ovary syndrome. Eur J Obstet Gynecol Reprod Biol. 2004; 113(2):214-20. https://doi.org/10.1016/j.ejogrb.2003.09.036.

62. Schachter M, Raziel A, Strassburger D, Rotem C, Ron-el R, Friedler S. Prospective, randomized trial of metformin and vitamins for the reduction of plasma homocysteine in insulin-resistant polycystic ovary syndrome. Fertil Steril. 2007;88(1):227-30. https://doi.org/10.1016/j.fertnstert.2006.11.071.

63. Sturrock ND, Lannon B, Fay TN. Metformin does not enhance ovulation induction in clomiphene resistant polycystic ovary syndrome in clinical practice. Br J Clin Pharmacol. 2002;53(5):469-73. https://doi.org/10.1046/j.13 65-2125.2002.01575.x.

64. Swanton A, Lighten A, Granne I, et al. Do women with ovaries of polycystic morphology without any other features of PCOS benefit from short-term metformin co-treatment during IVF? A double-blind, placebo-controlled, randomized trial. Hum Reprod. 2011;26(8):2178-84.

65. Tang T, Glanville J, Orsi N, et al. The use of metformin for women with PCOS undergoing IVF treatment. Hum Reprod. 2006;21(6):1416-25.

66. Tasdemir S, Ficicioglu C, Yalti S, Gurbuz B, Basaran T, Yildirim G. The effect of metformin treatment to ovarian response in cases with PCOS. Arch Gynecol Obstet. 2004;269(2):121-4. https://doi.org/10.1007/s00404-002-0447-8.

67. Vandermolen DT, Ratts VS, Evans WS, Stovall DW, Kauma SW, Nestler JE. Metformin increases the ovulatory rate and pregnancy rate from clomiphene citrate in patients with polycystic ovary syndrome who are resistant to clomiphene citrate alone. Fertil Steril. 2001;75(2):310-5. https:// doi.org/10.1016/50015-0282(00)01675-7.

68. Wang $X H$, Wang JQ, Xu Y, Huang LP. Therapeutic effects of metformin and laparoscopic ovarian drilling in treatment of clomiphene and insulinresistant polycystic ovary syndrome. Arch Gynecol Obstet. 2015;291(5):108994. https://doi.org/10.1007/s00404-014-3486-z.

69. Wei Z, Cao Y, Cong L, Zhou P, Zhang Z, Li J. Effect of metformin pretreatment on pregnancy outcome of in vitro matured oocytes retrieved from women with polycystic ovary syndrome. Fertil Steril. 2008;90(4):114954. https://doi.org/10.1016/.jertnstert.2007.07.1385.

70. Zain MM, Jamaluddin R, Ibrahim A, Norman RJ. Comparison of clomiphene citrate, metformin, or the combination of both for first-line ovulation induction, achievement of pregnancy, and live birth in Asian women with polycystic ovary syndrome: a randomized controlled trial. Fertil Steril. 2009; 91(2):514-21. https://doi.org/10.1016/j.fertnstert.2007.12.002.

71. Rapaport R. Endocrinology and metabolism clinics of North America. Pediatric endocrinology. Preface. Endocrinol Metab Clin N Am. 2012;41(4):xv-xvi.

72. Munro MG. Uterine polyps, adenomyosis, leiomyomas, and endometrial receptivity. Fertil Steril. 2019;111(4):629-40. https://doi.org/10.1016/j. fertnstert.2019.02.008.

73. Piltonen $\pi$. Polycystic ovary syndrome: endometrial markers. Best Pract Res CLIN Obstet Gynaecol. 2016;37:66-79. https://doi.org/10.1016/j.bpobgyn.201 6.03.008.

74. Faure M, Bertoldo MJ, Khoueiry R, Bongrani A, Brion F, Giulivi C, et al. Metformin in reproductive biology. Front Endocrinol. 2018;9:675. https://doi. org/10.3389/fendo.2018.00675.

75. Taylor HS, Arici A, Olive D, Igarashi P. HOXA10 is expressed in response to sex steroids at the time of implantation in the human endometrium. J Clin Invest. 1998;101(7):1379-84. https://doi.org/10.1172/JCl1597.

76. Cermik D, Selam B, Taylor HS. Regulation of HOXA-10 expression by testosterone in vitro and in the endometrium of patients with polycystic ovary syndrome. J Clin Endocrinol Metab. 2003;88(1):238-43. https://doi. org/10.1210/jc.2002-021072.

77. Daftary GS, Troy PJ, Bagot CN, et al. Direct regulation of beta3-integrin subunit gene expression by HOXA10 in endometrial cells. Mol Endocrinol. 2002;16(3):571-9.

78. Xiong F, Xiao J, Bai Y, et al. Metformin inhibits estradiol and progesteroneinduced decidualization of endometrial stromal cells by regulating expression of progesterone receptor, cytokines and matrix metalloproteinases. Biomed Pharmacother. 2019;109:1578-85.

79. Gellersen B, Brosens JJ. Cyclic decidualization of the human endometrium in reproductive health and failure. Endocr Rev. 2014;35(6):851-905. https:// doi.org/10.1210/er.2014-1045.

80. Detaille D, Wiernsperger N, Devos P. Metformin interaction with insulinregulated glucose uptake, using the Xenopus laevis oocyte model expressing the mammalian transporter GLUT4. Eur J Pharmacol. 1999:377(1): 127-36. https://doi.org/10.1016/S0014-2999(99)00413-6. 
81. Harvey AJ. The role of oxygen in ruminant preimplantation embryo development and metabolism. Anim Reprod Sci. 2007;98(1-2):113-28. https://doi.org/10.1016/j.anireprosci.2006.10.008.

82. Craciunas L, Gallos I, Chu J, Bourne T, Quenby S, Brosens JJ, et al. Conventional and modern markers of endometrial receptivity: a systematic review and meta-analysis. Hum Reprod Update. 2019;25(2):202-23. https:// doi.org/10.1093/humupd/dmy044.

83. Bonilla-Musoles F, Raga F, Osborne NG, Castillo JC, Bonilla F. Endometrial receptivity: evaluation with ultrasound. Ultrasound Q. 2013;29(1):3-20. https://doi.org/10.1097/RUQ.0b013e318281b60a.

84. Chan JM, Sukumar Al, Ramalingam M, Ranbir Singh SS, Abdullah MF. The impact of endometrial thickness (EMT) on the day of human chorionic gonadotropin (hCG) administration on pregnancy outcomes: a 5-year retrospective cohort analysis in Malaysia. Fertil Res Pract. 2018;4(1):5. https:// doi.org/10.1186/s40738-018-0050-8.

85. Kasius A, Smit JG, Torrance HL, Ejjkemans MJC, Mol BW, Opmeer BC, et al. Endometrial thickness and pregnancy rates after IVF: a systematic review and meta-analysis. Hum Reprod Update. 2014;20(4):530-41. https://doi.org/1 0.1093/humupd/dmu011.

86. Nishihara S, Fukuda J, Ezoe K, Endo M, Nakagawa Y, Yamadera R, et al. Does the endometrial thickness on the day of the trigger affect the pregnancy outcomes after fresh cleaved embryo transfer in the clomiphene citratebased minimal stimulation cycle? Reprod Med Biol. 2020;19(2):151-7. https://doi.org/10.1002/rmb2.12315.

87. Ding HF, Tian L. Relationship between endometrial thickness and pregnancy outcomes based on frozen-thawed embryo transfer cycles. Zhonghua Fu Chan Ke Za Zhi. 2018;53(11):742-8. https://doi.org/10.3760/ cma.j.jissn.0529-567x.2018.11.003.

88. Cacciatore B, Simberg N, Fusaro P, Tiitinen A. Transvaginal Doppler study of uterine artery blood flow in in vitro fertilization-embryo transfer cycles. Fertil Steril. 1996;66(1):130-4. https://doi.org/10.1016/S0015-0282(16)58400-3.

89. Al-Obaidi MT, Ali ZH, Al-Saadi WI, et al. Impact of letrozole versus clomiphene citrate on endometrial receptivity in Iraqi women with polycystic ovarian syndrome. J Clin Pharm Ther. 2019;44(4):618-22. https:// doi.org/10.1111/jcpt.12831.

90. Silva Martins R, Helio Oliani A, Vaz Oliani D, Martinez de Oliveira J. Subendometrial resistence and pulsatility index assessment of endometrial receptivity in assisted reproductive technology cycles. Reprod Biol Endocrinol. 2019;17(1):62. https://doi.org/10.1186/s12958-019-0507-6.

91. Schild RL, Knobloch C, Dorn C, Fimmers R, van der Ven H, Hansmann M. Endometrial receptivity in an in vitro fertilization program as assessed by spiral artery blood flow, endometrial thickness, endometrial volume, and uterine artery blood flow. Fertil Steril. 2001;75(2):361-6. https://doi.org/10.1 016/S0015-0282(00)01695-2.

92. Zhang T, He Y, Wang Y, Zhu Q, Yang J, Zhao X, et al. The role of threedimensional power Doppler ultrasound parameters measured on $\mathrm{hCG}$ day in the prediction of pregnancy during in vitro fertilization treatment. Eur J Obstet Gynecol Reprod Biol. 2016;203:66-71. https://doi.org/10.1016/j. ejogrb.2016.05.016.

93. Tsai HD, Chang CC, Hsieh YY, Lee CC, Lo HY. Artificial insemination. Role of endometrial thickness and pattern, of vascular impedance of the spiral and uterine arteries, and of the dominant follicle. J Reprod Med. 2000;45(3):195-200.

\section{Publisher's Note}

Springer Nature remains neutral with regard to jurisdictional claims in published maps and institutional affiliations.

Ready to submit your research? Choose BMC and benefit from:
- fast, convenient online submission
- thorough peer review by experienced researchers in your field
- rapid publication on acceptance
- support for research data, including large and complex data types
- gold Open Access which fosters wider collaboration and increased citations
- maximum visibility for your research: over 100M website views per year
At BMC, research is always in progress.
Learn more biomedcentral.com/submissions

